\title{
Aeroelastic Flight Data Analysis with the Hilbert-Huang Algorithm
}

\author{
Marty Brenner* \\ NASA Dryden Flight Research Center, Edwards, CA 93523, USA \\ Chad Prazenica ${ }^{\dagger}$ \\ University of Florida, Shalimar, FL 32579, USA
}

\begin{abstract}
This paper investigates the utility of the Hilbert-Huang transform for the analysis of aeroelastic flight data. It is well known that the classical Hilbert transform can be used for time-frequency analysis of functions or signals. Unfortunately, the Hilbert transform can only be effectively applied to an extremely small class of signals, namely those that are characterized by a single frequency component at any instant in time. The recently-developed Hilbert-Huang algorithm addresses the limitations of the classical Hilbert transform through a process known as empirical mode decomposition. Using this approach, the data is filtered into a series of intrinsic mode functions, each of which admits a well-behaved Hilbert transform. In this manner, the Hilbert-Huang algorithm affords time-frequency analysis of a large class of signals. This powerful tool has been applied in the analysis of scientific data, structural system identification, mechanical system fault detection, and even image processing. The purpose of this paper is to demonstrate the potential applications of the Hilbert-Huang algorithm for the analysis of aeroelastic systems, with improvements such as localized/online processing. Applications for correlations between system input and output, and amongst output sensors, are discussed to characterize the time-varying amplitude and frequency correlations present in the various components of multiple data channels. Online stability analyses and modal identification are also presented. Examples are given using aeroelastic test data from the F/A-18 Active Aeroelastic Wing aircraft, an Aerostructures Test Wing, and pitch-plunge simulation.
\end{abstract}

\section{Introduction}

The Hilbert transform is a classical tool that has been used in the structural dynamics community as an indicator of nonlinearity. It has also been used to estimate nonlinear damping and stiffness functions for single degree-of-freedom systems. The Hilbert transform expresses a signal as a harmonic with time-dependent frequency and amplitude. In this respect, it is an ideal tool for the analysis of nonstationary data. Unfortunately, the Hilbert transform has several shortcomings that limit its usefulness in practice. Most notably, the Hilbert transform computes a single instantaneous frequency for a signal at each instant in time. Therefore, when applied to a multi-component signal (i.e., a signal from a system with multiple modes), the Hilbert transform computes an instantaneous frequency that corresponds to a weighted average of the component frequencies. Such an instantaneous frequency does not provide any information as to the values of the individual component frequencies. ${ }^{4} \mathrm{~A}$ further limitation is that the Hilbert transform yields grossly distorted estimates of the frequency when applied to signals with nonzero mean and signals which have more extrema than zero crossings.

In order to address these shortcomings, an Empirical Mode Decomposition (EMD) was developed by Huang et al. ${ }^{8,9}$ as a means of decomposing a signal into a series of components known as Intrinsic Mode Functions (IMFs).

\footnotetext{
*Aerospace Engineer, Aerostructures Branch, Email: Martin.J.Brenner@nasa.gov.

$\dagger$ Visiting Assistant Professor, Graduate Engineering and Research Center, Member AIAA, Email: prazenic@ufl.edu.
} 
These IMFs are computed based on local characteristics of the signal and can be viewed as an adaptive, data-dependent basis. The IMFs form a complete, nearly orthogonal set of basis functions. Most importantly, each IMF contains only a single frequency component at any instant in time and therefore admits a well-behaved Hilbert transform. Taken collectively, the Hilbert spectra of the IMFs yield complete time-frequency information about the original signal. This approach, which has been termed the Hilbert-Huang Transform (HHT), makes it possible to apply the Hilbert transform to an extremely general class of functions and signals.

Although a relatively new tool, the Hilbert-Huang transform algorithm has received considerable attention in a number of engineering disciplines. This HHT algorithm has been applied in the analysis of scientific data, ${ }^{8-10}$ structural system identification, ${ }^{26-28}$ and mechanical system fault detection. ${ }^{11,15,25,29}$ A recent adoption into the image processing field, ${ }^{13,14,16}$ the two-dimensional EMD is an adaptive image decomposition without the limitations from filter kernels or cost functions. The IMFs are interpreted as spatial frequency subbands with varying center frequency and bandwidth along the image. The EMD is a truly empirical method, not based on the Fourier frequency approach but related to the locations of extrema points and zero crossings. Based on this, the concept of "empiquency," used for time or space and short for "empirical mode frequency," was adopted to describe signal (image) oscillations based on the reciprocal distance between two consecutive extrema points. High concentrations of extrema points have high empiquency with sparse areas having low empiquency. Hence, applications for time-frequency-space signal processing are feasible.

A problem with the very versatile and most commonly used Morlet wavelet in dynamics data analysis is its leakage generated by the limited length of the basic wavelet function, which makes the quantitative definition of the energyfrequency-time distribution difficult. The interpretation of the wavelet can also be counterintuitive. For example, definition of a local event in any frequency range requires analysis in the high-frequency range, for the higher the frequency the more localized the basic wavelet. A local event in the low-frequency range requires an extended period of time to discern it. Such interpretation can be difficult if possible at all. Another problem with wavelet analysis is its nonadaptive nature. Wavelet basis functions are predefined, whether of one type, or a multi-wavelet basis, or a dictionary of wavelets is selected. Data analysis is then constrained to these bases. Since the Morlet wavelet is Fourier based, it also suffers the many shortcomings of Fourier spectral analysis. A truly adaptive basis is a necessary requirement for nonstationary and nonlinear time series analysis and should be based on and derived from the data.

In this paper, results are obtained from the new approach. With the HHT, the intrinsic mode functions yield instantaneous frequencies as functions of time that give sharp identification of imbedded structures. The main conceptual innovation in this approach is the introduction of the instantaneous frequencies for complicated data sets, which eliminate the need of spurious harmonics to represent nonlinear and nonstationary signals. This paper looks at the effect of enhancements like local/on-line versions of the algorithm. ${ }^{20}$ To date, HHT analysis has only been performed on individual signals without regard to correlation with other data channels, or system inputs-to-outputs. Application for correlations between system signals are introduced to characterize the time-varying amplitude and frequency modulations present in the various components of multiple data channels including input and distributed sensors. In these respects, this paper attempts to elucidate the way EMD behaves in the analysis of F/A-18 Active Aeroelastic Wing (AAW) aircraft, ${ }^{18}$ aeroelastic and aeroservoelastic flight test data as well as Aerostructures Test Wing ${ }^{12}$ and pitch-plunge simulation data.

\section{Empirical Mode Decomposition}

The classical Hilbert transform is, in principle, an effective tool for time-frequency analysis. Unfortunately, in practice, it can only be applied to an extremely restricted class of signals. In order to extend the utility of the Hilbert transform to more general signals, Huang et al. ${ }^{8,9}$ developed the empirical mode decomposition (EMD) as a means of preprocessing data before applying the Hilbert transform. The EMD procedure decomposes the original signal into a set of intrinsic mode functions (IMFs), each of which admits a well-behaved Hilbert transform. The Hilbert transform is then applied to each individual IMF, yielding an instantaneous frequency and amplitude for each IMF. Therefore, this procedure, termed the Hilbert-Huang transform (HHT), enables the Hilbert transform to be applied to multi-component signals. 
There are two criteria that each IMF must satisy in order to be amenable to the Hilbert transform. Namely, each IMF must have zero mean and the number of local extrema and zero crossings in each IMF can differ by no more than one. The first step in the EMD procedure is to connect all the local maxima of the original signal using a cubic spline. Similarly, the local minima are also connected with a cubic spline. The two splines define the envelope, and the mean of this envelope is then calculated and subtracted from the original signal. The resulting signal is then tested to see if it satisfies the criteria for an IMF. If it does not, the sifting process is repeated until a suitable first IMF, $c_{1}(t)$, is obtained. The sifting process is then applied to the residual signal $x(t)-c_{1}(t)$ to obtain the next IMF. This process is repeated until all that is left is a final residual, $r(t)$, which represents the trend in the data and is not an IMF. Therefore, as shown in Eq. 1, the EMD procedure yields a decomposition of the original signal in terms of $n$ IMFs and the residual:

$$
x(t)=\sum_{j=1}^{n} c_{j}(t)+r(t)
$$

The number of IMFs obtained is dependent on the original signal.

The EMD procedure serves to generate IMFs that are amenable to the Hilbert transform. In particular, the manner in which the EMD is performed guarantees that each IMF will only possess a single harmonic at any instant in time. Currently, the EMD procedure is ad hoc in the sense that there is no rigorous mathematical theory behind it. Recent attempts have been made to formalize EMD, most notably the work of those who explored the properties of B-splines and their use in the EMD process. Despite the lack of a firm theoretical foundation, several mathematical properties of the IMFs are well understood. In particular, it is clear from Eq. 1 that the IMFs, along with the residual, form a complete basis for the original signal $x(t)$. In addition, Huang et al. ${ }^{8}$ demonstrated that the IMFs are nearly orthogonal with an orthogonality index $\left(O I_{m n}\right.$ defined in Eq. 2$)$ for the IMFs. This definition seems to be global but actually only applies locally. Adjacent IMFs could have data with the same frequency but at different times.

$$
O I_{m n}=\sum_{t=0}^{t=T} \sum_{j=1}^{m} \sum_{k(\neq j)=1}^{n} \frac{c_{j}(t) c_{k}(t)}{x(t)^{2}}
$$

The EMD does not yield a unique basis for the original signal since there are countless sets of suitable IMFs that can be generated from a given signal. However, the various IMF sets from the different sifting criteria are all equally valid representations of the data provided their orthogonality indices are sufficiently small. ${ }^{8,10}$ The IMFs depend on the stoppage criterion, maximum number of siftings, intermittance criteria, the end point boundary conditions, and use of curvature- or extrema-based sifting. ${ }^{8-10}$ The uniqueness problem can only be meaningful if all these parameters are fixed a priori. The problem is how to optimize the sifting procedure to produce the "best" IMF set. These questions are difficult to answer theoretically. In Huang et. al., ${ }^{10}$ a confidence limit is defined for the first time without invoking the ergodic assumption. This provides a stable range of stopping criteria for the EMD-sifting operation, thereby making the HHT method more definitive. Discussion later points out that the EMD sifting process also acts in such a manner as to obtain IMFs that correspond to approximate bandpass filtering of the original signal.

Once the EMD procedure has been used to generate a set of IMFs, the Hilbert transform can be applied to each individual IMF. In this manner, an instantaneous frequency and amplitude is computed for each function. A common method for displaying the Hilbert spectrum is to generate a two-dimensional plot with time and frequency axes. The amplitude is then plotted as a color spectrogram in the time-frequency plane. By plotting the Hilbert spectra of all the IMFs together one obtains complete time-frequency information about the original signal.

To demonstrate the importance of the EMD in obtaining meaningful Hilbert spectra for general signals, consider the following signals:

$$
\begin{aligned}
& x_{1}(t)=\sin (2 \pi t)+\sin (8 \pi t)+\sin (32 \pi t) \\
& x_{2}(t)=\sin (4 \pi t)+t
\end{aligned}
$$

The signal $x_{1}$ is a combination of harmonics with frequencies of $\{1,4,16\} \mathrm{Hz}$ while the signal $x_{2}$ is composed of a ramp signal and a $2-\mathrm{Hz}$ sine wave. First, consider the Hilbert spectra obtained by directly applying the Hilbert 
transform to each signal. Figure 1 displays the resulting Hilbert spectra for both signals. The Hilbert spectrum of $x_{1}$ yields a fluctuating frequency with most of the energy concentrated at $7 \mathrm{~Hz}$. This corresponds to the average of the three frequencies present in $x_{1}$ (each of which has the same amplitude) and gives no useful information about the original signal. This averaging is due to the fact that the Hilbert transform computes a single instantaneous frequency for the signal. The Hilbert spectrum of $x_{2}$ illustrates the effect of a nonzero mean. In this case, the estimated frequency is distorted and the $2-\mathrm{Hz}$ harmonic is not identified at all.
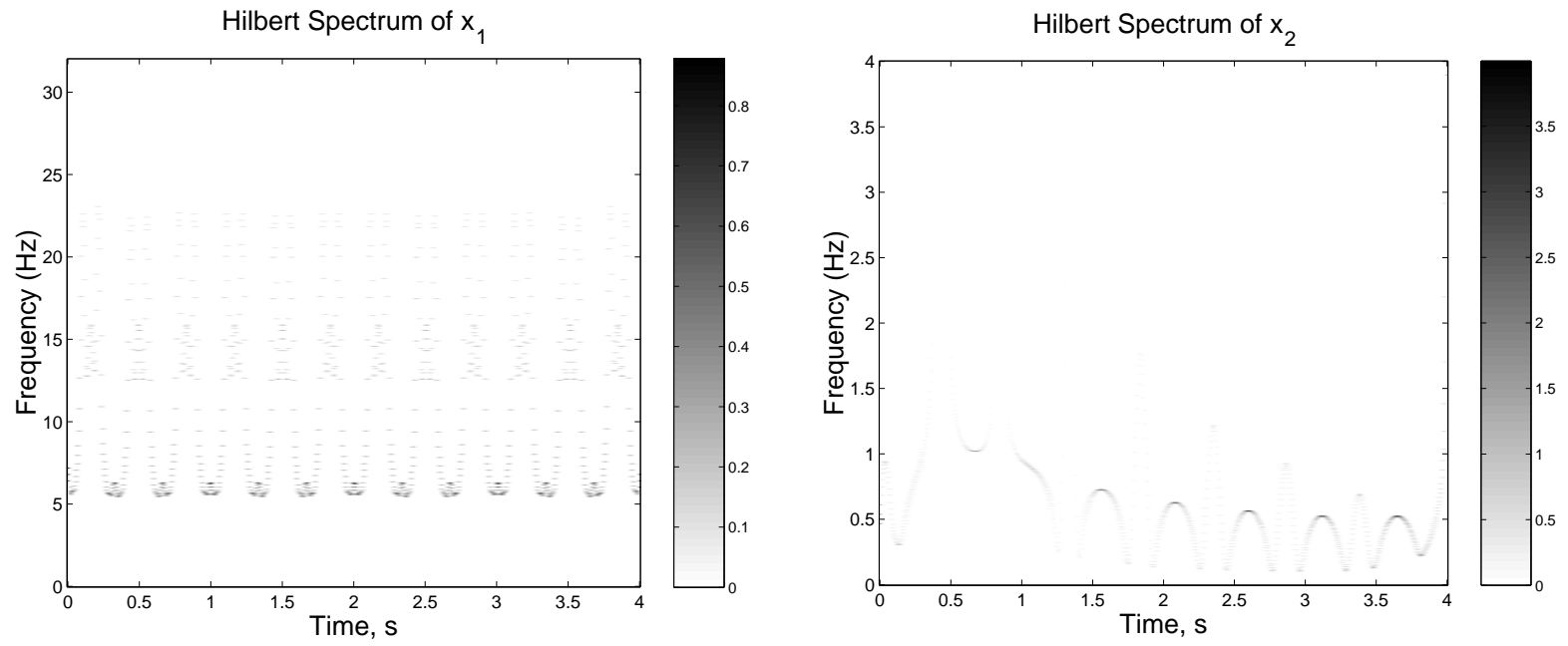

Figure 1. Time-frequency spectra from the Hilbert transforms of the signals $x_{1}$ and $x_{2}$.
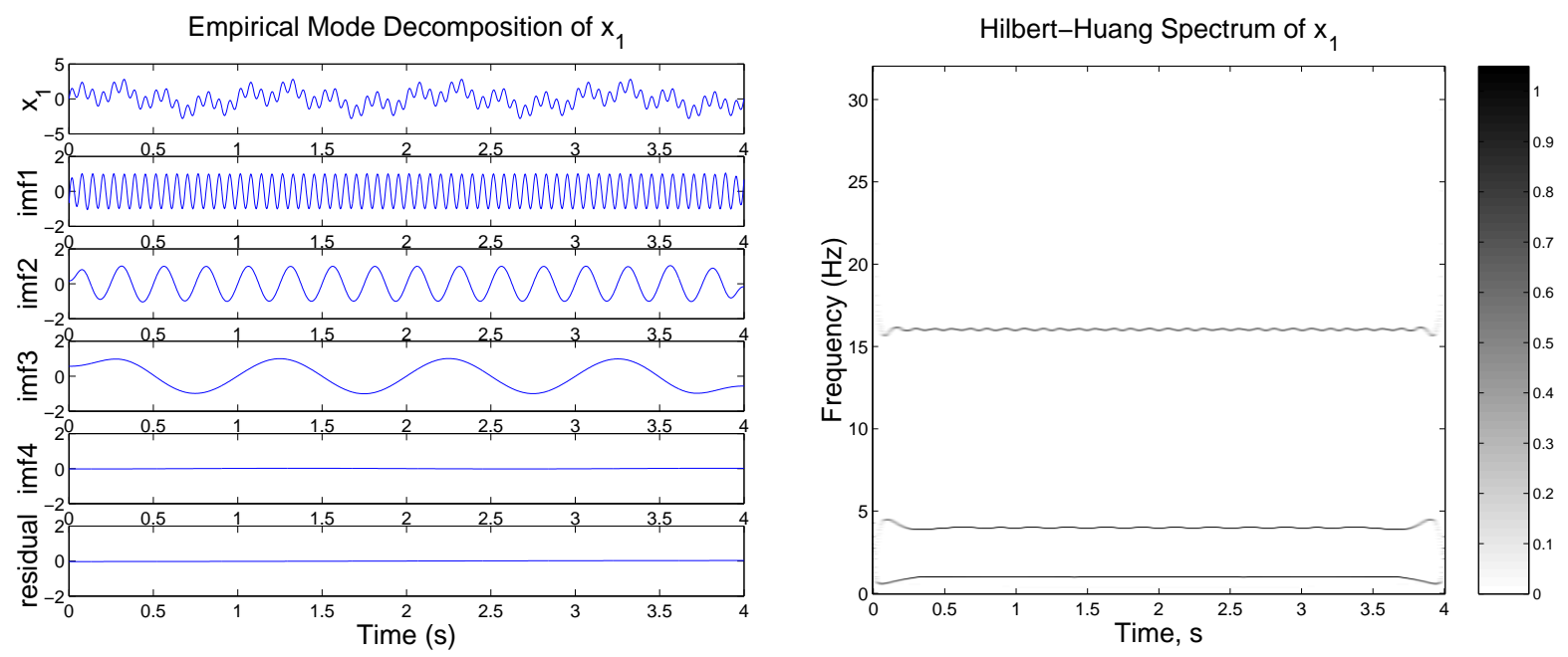

Figure 2. Empirical mode decomposition and the Hilbert-Huang spectrum of the signal $x_{1}$.

Figure 2 displays the signal $x_{1}$ and its decomposition into four IMFs (imf $1=c_{1}$, imf2 $=c_{2}$, etc.) and a residual. Clearly, each IMF has captured a different frequency component of the original signal. The fourth IMF is extremely small and results from boundary effects in the EMD process. The Hilbert spectra of the individual IMFs are plotted together in Fig. 2. Now, an effective Hilbert spectrum, or Hilbert-Huang spectrum, of $x_{1}$ has been obtained, with all three frequency components clearly identified. Similarly, Fig. 3 depicts two IMFs and a residual generated from 

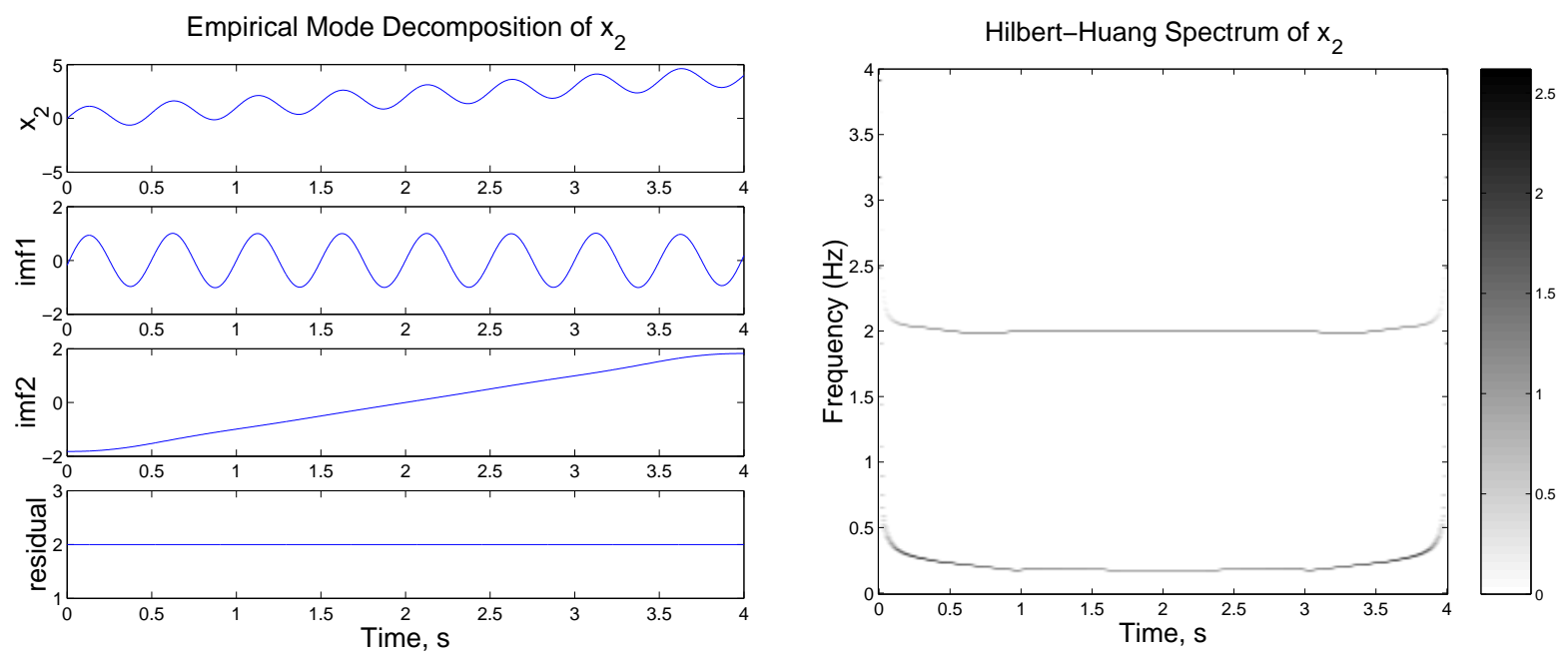

Figure 3. Empirical mode decomposition and the Hilbert-Huang spectrum of the signal $x_{2}$.

the signal $x_{2}$. The $2-\mathrm{Hz}$ frequency component has been sifted into the first IMF and the ramp component has been identified as the second IMF. The mean of the signal is 2, which has been separated out as the residual. The Hilbert spectrum clearly shows the $2-\mathrm{Hz}$ component and estimates a low frequency for the ramp component. This occurs because the ramp is treated as part of a low frequency wave. The IMFs and Hilbert spectra in Figs. 2 and 3 illustrate that there are some minor boundary effects associated with the EMD process. Most importantly, these examples demonstrate that EMD makes it possible to apply the Hilbert transform to signals that otherwise do not admit wellbehaved Hilbert transforms.
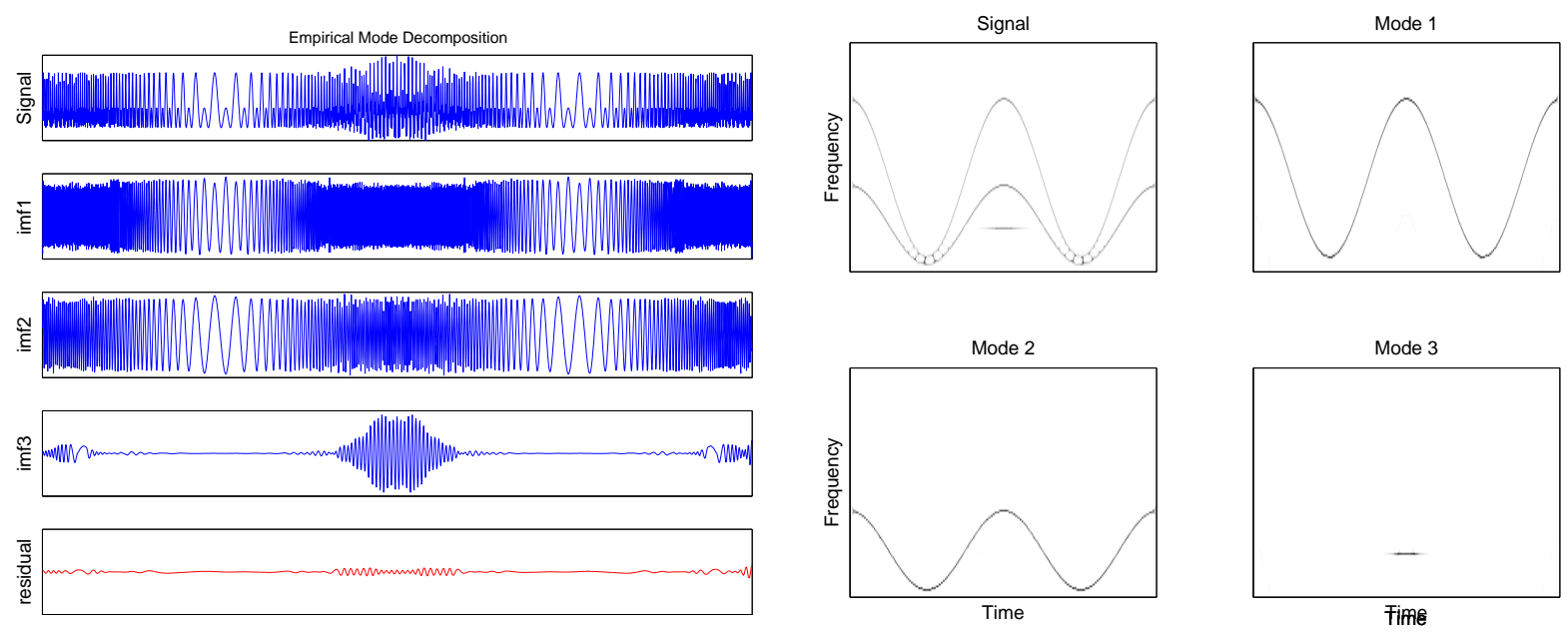

Figure 4. Empirical mode decomposition (EMD) and the Hilbert-Huang spectrum of the sum of two sinusoidal frequency modulations (FMs) and one Gaussian wave packet. Original signal (top), FM1, FM2, Gaussian wave packet, and residual.

Finally, two more examples ${ }^{20}$ illustrate automatic and adaptive (signal-dependent) time-variant filtering of general mixtures of signals. A signal composed of three components which significantly overlap in time and frequency is 

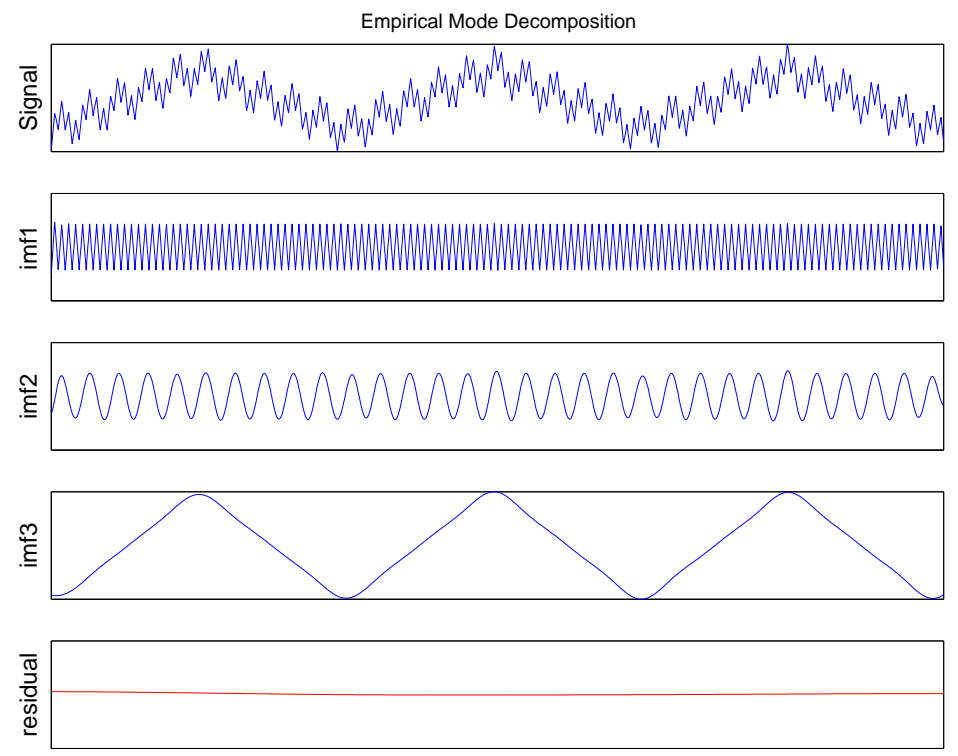

Figure 5. Empirical mode decomposition of the sum of one sinusoid with two triangular waveforms. Original signal (top), high-frequency triangular, sinusoid, low-frequency triangular, and residual.

successfully decomposed in Fig. 4 for the sum of two sinusoidal frequency modulations and one Gaussian wave packet. Another example, accenting the nonharmonic nature of EMD, is given in Fig. 5. The analyzed signal (top) is the sum of three components, a sinusoid superimposed on two triangular waveforms with periods smaller and larger than the sinusoid. The decomposition performed by the EMD is given in the three IMFs and the residue. In this case, both linear (sinusoid) and nonlinear (triangular) oscillations are effectively identified and separated. Harmonic analysis (Fourier, Morlet wavelets) would produce a less succinct and more nondescriptive decomposition.

\section{Filtering Properties}

The filtering properties of EMD have been studied in some detail. ${ }^{6,7,24}$ The EMD process yields a data-dependent decomposition that focuses on local characteristics of the signal. In particular, EMD sifts out the highest-frequency component in the signal at any given time. Indeed, EMD has surprisingly been shown to behave as a dyadic bandpass filter when decomposing Gaussian white noise, much like a multiresolution wavelet decomposition. However, the EMD method as an equivalent dyadic filter bank is only in the sense of its global behavior over the entire time extent. In representing the time-frequency distribution, the Hilbert spectrum of each IMF is actually localized at any time. This is different from predetermined filtering such as with Morlet wavelet filtering.

\section{Analytical Interpretation}

The EMD is faced with the fundamental difficulty of not admitting an analytical definition, but of rather being defined by the algorithm itself, thus making the analysis of its performance and limitations difficult. The need for rigorous mathematical foundation is imperative. This fundamental problem of the empirical mode decomposition has to be resolved since only with the intrinsic mode function can nonlinear distorted waveforms be resolved from nonlinear processes. There have been attempts to circumvent the mathematical difficulties in the EMD with some success by casting the IMFs in terms of B-splines, ${ }^{3}$ and applying towards mechanical system fault-detection. ${ }^{15}$ System identification of the IMFs as a multi-component system is suggestive in the light of multiresolution system identification procedures such as multiresolution singular value decompositions, Kalman filters, and subspace algorithms. 
More significantly, characterization of IMFs as solutions to certain self-adjoint ordinary differential equations is demonstrated. ${ }^{21,22}$ Construction of envelopes which do not rely on the Hilbert transform is used directly to compute the coefficients of the differential equations. These equations are natural models for linear vibrational problems and provide further insight into both the EMD procedure and utilizing its IMF components to identify systems of differential equations naturally associated with the components. One of the uses of the EMD procedure is to study solutions to differential equations, and vibration analysis was a major motivation in the development of the Sturm-Liouville theory.

\section{Hilbert Transform and Instantaneous Frequency}

The Hilbert transform of a time-domain function or signal $x$ is defined in Eq. 3,

$$
y(t)=\mathrm{H}\{x(t)\}=\frac{1}{\pi} \mathrm{PV} \int_{-\infty}^{\infty} \frac{x(\tau)}{t-\tau} d \tau
$$

where PV denotes the Cauchy principal value, needed because the integrand is singular at $\tau=t$. The Hilbert transform can be viewed as the convolution of the original signal with $1 / t$, emphasizing temporal locality of $x(t)$. Note that, unlike Fourier analysis, the Hilbert transform of a time-domain signal is another time-domain signal. The Hilbert transform is sometimes applied to frequency-domain signals using a similar expression as Eq. 3, but this paper will focus on the time-domain case. In practice, the Hilbert transform is usually calculated using the Fourier transform. Therefore, the fast Fourier transform algorithm can be employed for the efficient calculation of the Hilbert transform.

A signal, $x$, and its Hilbert transform, $y$, can be used to define a complex analytical signal as in Eq. 4.

$$
z(t)=a(t) \mathrm{e}^{i \theta(t)}=x(t)+i y(t)
$$

Therefore, the Hilbert transform pair $\{x(t), y(t)\}$ can be expressed as a harmonic function with time-varying amplitude $a(t)$ and time-varying phase angle $\theta(t)$.

$$
\begin{aligned}
& a(t)=\sqrt{x(t)^{2}+y(t)^{2}} \\
& \theta(t)=\tan ^{-1}\left(\frac{y(t)}{x(t)}\right)
\end{aligned}
$$

Given the time-dependent phase angle, the instantaneous frequency of the signal can be defined as ${ }^{5}$

$$
\omega(t)=\frac{d \theta(t)}{d t} .
$$

In the context of Eq. 3 with instantaneous frequency, the Hilbert transform of an IMF can be interpreted as giving the best fit with a sinusoidal function to the data weighted by $1 / t$. Instantaneous frequency can be computed using the derivative definition or centralized finite difference. ${ }^{5}$ In general, there are an infinite number of ways to express a signal as in Eq. 4, so there can also be an infinite number of instantaneous frequencies. The Hilbert-transform pair was proposed to uniquely define the amplitude and phase by building the complex analytic signal from the given signal with the original signal, $x(t)$, as the real part and the orthogonal transformed signal, $y(t)$, the imaginary part, out of phase with $x(t)$ by $\frac{\pi}{2}$. Note that Fourier analysis yields the decomposition

$$
x(t)=\sum_{j=0}^{\infty} a_{j} e^{i \omega_{j} t}
$$

which is similar to the form of the Hilbert transform in Eq. 4. A key difference, however, is that the Fourier decomposition is in terms of harmonics with constant amplitudes and frequencies. In contrast, the Hilbert transform yields instantaneous amplitudes and frequencies. Therefore, in principle, the Hilbert transform is an ideal tool for the time-frequency analysis of a general class of signals, including nonstationary signals.

7 of 29 
Another important distinction between Fourier analysis and the Hilbert transform is that the Fourier decomposition is in terms of multiple harmonics of constant amplitude and frequency, thereby producing artificial harmonics to maintain energy conservation for nonstationary and nonlinear data. In contrast, the Hilbert transform of a signal yields an expression in terms of a single harmonic with a time-varying frequency and amplitude. For this reason, the Hilbert transform is only suitable for the analysis of mono-component signals, or signals that are composed of a single frequency component at any instant in time. This is a considerable limitation as it implies that the Hilbert transform cannot be directly applied to signals that are composed of multiple harmonics. As was shown, the Hilbert transform fails to identify the individual frequencies and instead computes a single instantaneous frequency that corresponds to a weighted average of the component frequencies. The resulting instantaneous frequency is both physically invalid and erroneous ${ }^{4}$ since a multi-component signal has more than one instantaneous frequency. An additional limitation of the Hilbert transform is that it yields extremely distorted estimates of the instantaneous frequencies of signals with nonzero mean and signals that have more local extrema than zero crossings.

The EMD responds to the dilemma surrounding the applicability of instantaneous frequency. It decomposes a multi-component signal into its associated mono-components while not obscuring or obliterating the physical essentials of the signal and allows the traditional definition of instantaneous frequency to be complete by being applicable to signals of both mono- and multi-component. To follow the true frequency evolution within a multi-component signal, it is necessary to break down the components into individual and physically meaningful intrinsic parts. The adaptive and nonarbitrary decomposition using EMD produces an orthogonal set of intrinsic components each retaining the true physical characteristics of the original signal. The mono-components or intrinsic modes satisfy the conditions for a well-defined notion of instantaneous frequency. These conditions include symmetry, no dependence on predefined time scales, revelation of the nature of simultaneous amplitude and phase variation, and near-orthogonality.

Finally, since instantaneous frequency displays frequency variation with time, changes of dynamic states indicative of nonlinearity can be identified. For example, if the instantaneous frequency of a new mode is about half of the frequency of the old mode in a bifurcation, period doubling occurs. If the instantaneous frequency of the new mode is disproportionate with the old mode, quasi-periodic bifurcation occurs. Similarly, intermittence and chaotic motion can be determined. A dynamic state can be diagnosed simultaneously by observing the changes in time of the instantaneous frequency components and their corresponding energy. In summary, the concept of mode defined in relation to instantaneous frequency as a periodic-modal structure in the instantaneous time-frequency plane is found to be more appropriate than artificial sinusoidal harmonics in characterizing nonlinear responses. ${ }^{25}$ Instantaneous frequency is a quantity critical for understanding nonstationary and nonlinear processes.

\section{Local On-Line Decompositions}

In the original EMD formulation, sifting iterations are applied to the predefined full length signal as long as there exists a locality at which the mean of the upper and lower envelopes is not considered sufficiently small enough. Excessive iterations on the entire signal to achieve a better local approximation contaminates other parts of the signal by overcompressing the amplitude and overdecomposing it by spreading out its components over adjacent intrinsic modes, i.e., overiteration leads to overdecomposition. The various stoppage criteria (to fulfill that the number of extrema and the number of zero crossings must differ at most by one, and that the mean between the upper and lower envelopes must be close to zero) are attempts to avoid the rigor of the symmetry of the envelopes without a mathematically rigorous definition for an adaptive basis. The hierarchical and nonlinear nature of the EMD algorithm will not provide that the EMD of segmented signals will be the segmentation of individual EMDs. Therefore, a variation referred to as local $\mathrm{EMD}^{20}$ introduces an intermediate step in the sifting process. Localities at which the error remains large are identified and isolated, and extra iterations are applied only to them. This is achieved by introducing a weighting function such that maximum weighting is on those connected segments above a threshold amplitude, with a soft decay to zero outside those supports, much like soft-thresholding is done in wavelet denoising.

Another option is based on the idea that sifting relies on interpolations between extrema, and thus only requires a finite number of them (five minima and five maxima in the case of the recommended cubic splines ${ }^{3,8}$ ) for local interpolation. Extraction of a mode could therefore be moving blockwise instead of globally over the entire time span.

8 of 29

American Institute of Aeronautics and Astronautics 
This led to the development of the on-line EMD. ${ }^{20}$ A prerequisite for the sliding window extraction of a mode is to apply the same number of sifting steps to all blocks in order to prevent possible discontinuities. Since this would require the knowledge of the entire signal, the number of sifting operations is proposed to be fixed a priori to a number less than 10 for effective application of the on-line version of the EMD algorithm operating in coordination with the local EMD described above. The leading edge of the window progresses when new data become available, whereas the trailing edge progresses by blocks when the stopping criterion is met on a block. Therefore, an IMF and corresponding residual are computed sequentially, then again applied to this residual, thus extracting the next mode with some delay.

An aileron command multisine input used on the F/A-18 AAW for aeroservoelastic response and flutter clearance is shown in Fig. 6 (top plots) using the standard EMD (left) and local/on-line version (right). The bandpass nature of IMFs $6,7,24$ is reflected in the three standard IMF mean frequencies, $\{23.9,16.3,7.7\} \mathrm{Hz}$ for each of IMFs $\{\# 1, \# 2, \# 3\}$, respectively, and on-line corresponding IMF mean frequencies $\{23.6,13.0,7.2\} \mathrm{Hz}$. Immediately noticeable is the more efficient extraction of the signal components by the local/on-line algorithm, most evident by the sparse second and third IMFs (imf2 and imf3) being more sparse than the corresponding standard IMFs. Besides the obvious advantage of an on-line algorithm for decomposing data, it has been found to clearly surpass the standard global algorithm in terms of computational burden, especially with long original data records. An added bonus is that it generally has better orthogonality properties among the IMFs, witnessed by an order of magnitude improvement in the orthogonality index defined in Eq. 2.
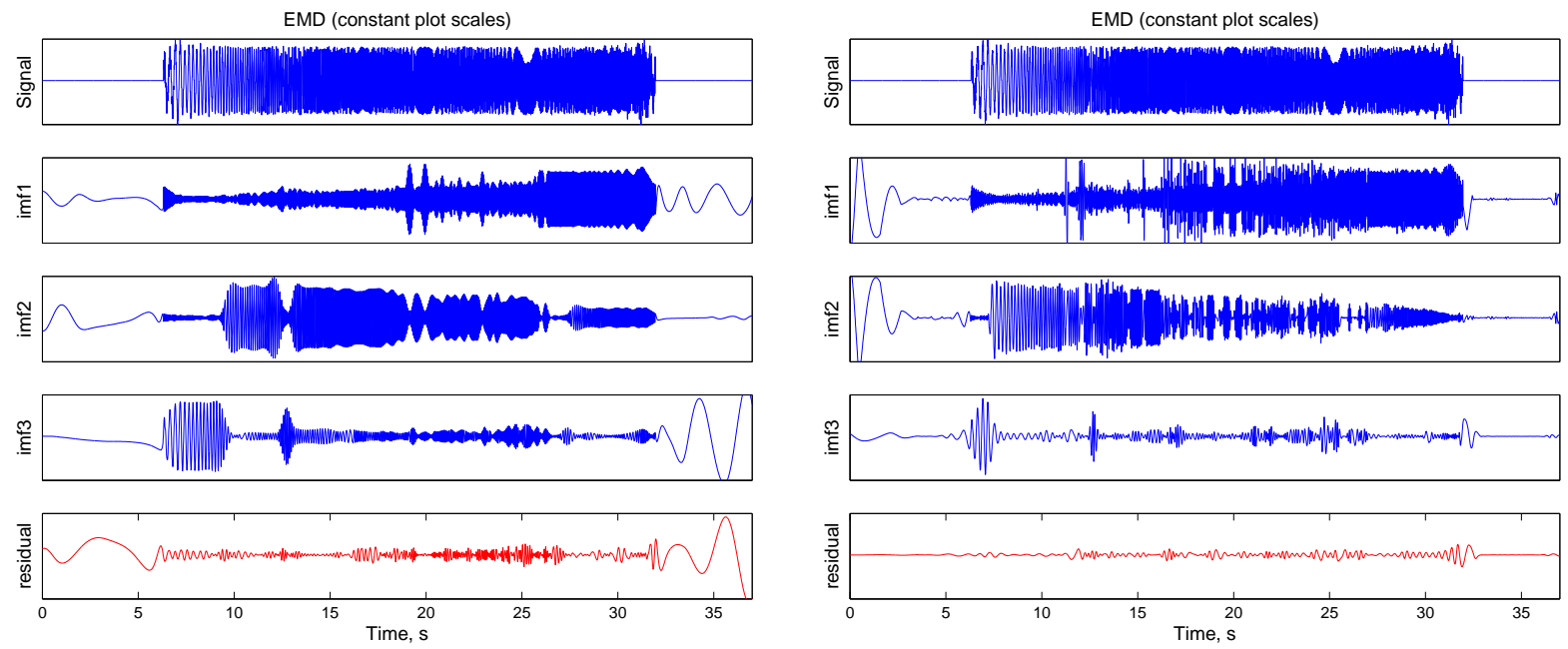

Figure 6. Standard (left) and local/on-line (right) empirical mode decomposition of an F/A-18 AAW multisine aileron command input, with original signal at top and residual at bottom.

\section{Local Analytic Signal Correlation}

Since the IMFs allow permissible, meaningful, physically sensible, and unique interpretations of instantaneous frequency of general signals of interest, they fit into the class of asymptotic signals such that the time variation of the IMF amplitude and frequency may directly be recovered from the time variation of the amplitude and of the phase derivative of the associated analytic signal. An IMF after performing the Hilbert transform can be written as in Eq. 4. These complex components are now used for analysis of analytic data correlations ${ }^{1,2}$ between input-output and amongst spatially distributed sensor outputs. Note that these analyses are all local in nature since there are no assumptions of stationarity, and differ from classical double-time expressions ${ }^{1}$ by being instantaneous. 


\section{Local Correlation Coefficient}

Correlations are made between transformed IMFs of various signals given the associated complex analytic signals

$$
\begin{aligned}
Z_{x}(t) & =A_{x}(t) e^{i \phi_{x}(t)}=x(t)+i x_{H}(t) \\
Z_{y}(t) & =A_{y}(t) e^{i \phi_{y}(t)}=y(t)+i y_{H}(t)
\end{aligned}
$$

by considering the cross-analytic signal defined in Eq. 5 by

$$
Z_{x y}(t)=Z_{x}^{*}(t) Z_{y}(t)=A_{x}(t) A_{y}(t) e^{i \phi_{x y}(t)}=A_{x}(t) A_{y}(t) e^{i\left[\left(\omega_{y}-\omega_{x}\right) t+\left(\phi_{0 y}-\phi_{0 x}\right)\right]}
$$

which is not necessarily analytic but can be used to characterize the time variation of the phase difference between components at similar frequencies extracted from two simultaneously acquired signals. This Hilbert demodulation technique is only applicable for analytic components, in this case the transformed IMFs of the original signals. If the two components are mainly in-phase and a change occurs in the cross-analytic signal, the instantaneous frequencies of the two components may be drifting apart and this produces an instantaneous contribution to the phase difference from Eq. 5. A measure of the local correlation between components, in terms of simultaneous changes in instantaneous amplitude or frequency (phase) between analytic signals, is the Hilbert Local Correlation Coeffcient, $H L C C$.

$$
H L C C(t)=\frac{R e\left[Z_{x y}\right]}{\left|Z_{x y}\right|}=\cos \left[\phi_{x y}(t)\right] .
$$

As a simple illustration of the utility of the $H L C C$, Fig. 7 shows the EMDs of wingtip accelerometer responses (accels) due to a symmetric aileron input, with the top plot in each set being the original signal, followed by EMDs 1-3 and the residual. The top two sets represent the forward (fwd) wingtip accels, and corresponding IMFs seem not to compare quite as well as the bottom two IMF sets of aft wingtip accels for the same input. The bottom IMF sets seem much more correlated with each other than the top two sets.

Table 1 lists the means, medians, and standard deviations of the $H L C C$ data over the time span of the maneuver. Note that $H L C C_{x y}^{m n}$ indicates correlation coefficients between Accel\#m and Accel\#n, using cross-analytic IMF function $Z_{x y}$ correlating Accel\#m's IMF $x$ to Accel\#n's IMFy. Results from the table indicate strongest Hilbert local correlation coefficients for $Z_{x y}$ when $x=y$, as expected since this is a correlation between similar bandpass characteristics of IMFs from different responses due to the same input. For comparison, the standard statistical signal correlation coefficient between Accel\#1 and \#3 is $C^{13}=0.8633$, and between Accel\#2 and \#4 is $C^{24}=0.8332$ (correlating fwd-to-fwd accels and aft-to-aft accels, respectively, wingtip-to-wingtip). This is consistent with the top original signal plots in Fig. 7 in that the forward wingtip accels (top two, fwd) seem to correlate reasonably well with each other, as also between the bottom two (aft), but not top compared with the bottom. Evident in Table 1 is the common standard deviations ( $S T D$ in all the correlations, near $S T D=0.7$ ). In the $H L C C$ values good commonality is found between the mean and medians comparing $H L C C^{13}$ and $H L C C^{24}$ in that the trend is similar amongst the cross-analytic IMF functions $Z_{x y}$ where $x$ and $y$ correspond to respective IMF numbers in different accel EMDs. This is surprising, since in Fig. 7 the correlation in corresponding IMFs seems much worse between the two fwd accels (top plot IMFs) than between the two aft accels (bottom plot IMFs).

Figure 8 shows the $H L C C$ functions between Accel\#1 and Accel\#3 $\left(H L C C_{x y}^{13}\right)$ at 5-20 s. Decomposing and representing correlations in this fashion allows a true time-localized instantaneous measure revealing subtle properties in the data and inconspicuous relations to other data sets using analytic components.

\section{Instantaneous Transfer Function}

In addition to investigating correlations between sensors, an instantaneous system transfer function is introduced with instantaneous magnitude and the $H L C C$ as a phase parameter between input-output analytic signals. This is viable in terms of the HHT inducing analytic properties to the IMFs to yield localized system input-output properties. Instantaneous transfer function $(I T F)$, its instantaneous magnitude $(I M)$, and its instantaneous phase $(I P)$ are defined.

$$
I T F(t)=\frac{Z_{x y}(t)}{Z_{x x}(t)} ; \quad I M(t)=|I T F(t)| ; \quad I P(t)=\cos ^{-1}[H L C C(t)]=\phi_{x y}(t)
$$



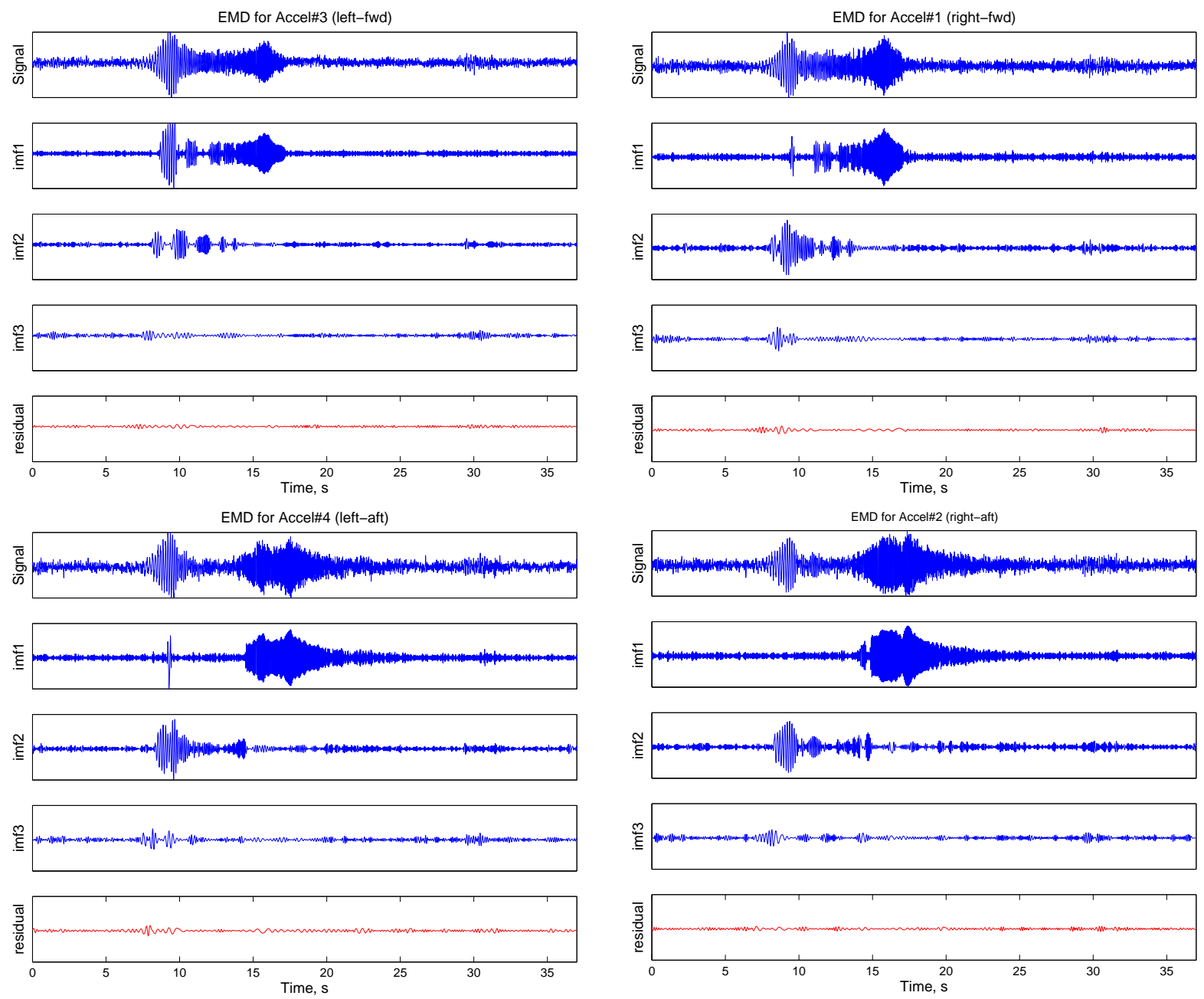

Figure 7. EMDs (with constant vertical plot scales) of F/A-18 AAW aircraft left-fwd wingtip accel (top left, Accel\#3), rightfwd wingtip accel (top right, Accel\#1), left-aft wingtip accel (bottom left, Accel\#4), and right-aft wingtip accel (bottom right, Accel\#2), responses from the multisine symmetric aileron command shown in Fig. 6 (original signal at top and residual at bottom in each set).

In Fig. 9 the top set of plots represent instantaneous magnitude $\left(I M_{x y}^{a 1}\right)$ and the bottom plots represent instantaneous phase $\left(I P_{x y}^{a 1}\right)$ from the instantaneous transfer function $\left(I T F_{x y}^{a 1}\right)$ of the aileron command input (Fig. 6) to Accel\#1 (top right, Fig. 7) analytic IMFs (Hilbert-transformed i.e., $\left\{Z_{x}, Z_{y}\right\}$ ). Note that $I T F_{x y}^{m n}$ indicates transfer functions between Input\#m and Accel\#n, using cross-analytic IMF function $Z_{x y}$ correlating Input\#m's IMF $x$ to Accel\#n's IMFy. In this sense, by interpreting the input-output Hilbert-transformed pairs of IMFs as a multi-component system of input-output signals, local stability measures are deemed to be feasible by tracking gain, phase, and instantaneous frequencies between each IMF pair. How these local IMF properties correspond to global system properties, given the analytic transformed IMFs, and aeroelastic and aeroservoelatic applications in stability and health monitoring, is currently being researched. 
Table 1. $H L C C$ results from F/A-18 AAW wingtip acceleration data.

\begin{tabular}{c|ccc|ccc}
$Z_{x y}$ & Mean $H L C C^{13}$ & Median $H L C C^{13}$ & $S T D^{13}$ & Mean $H L C C^{24}$ & Median $H L C C^{24}$ & $S T D^{24}$ \\
\hline$Z_{11}$ & 0.3283 & 0.5968 & 0.6801 & 0.2733 & 0.5309 & 0.7060 \\
$Z_{12}$ & 0.0917 & 0.1782 & 0.7171 & 0.0502 & 0.1080 & 0.7142 \\
$Z_{13}$ & 0.0098 & 0.0252 & 0.7113 & 0.0005 & -0.0106 & 0.7057 \\
$Z_{21}$ & 0.0953 & 0.1870 & 0.7148 & 0.0492 & 0.0896 & 0.7095 \\
$Z_{22}$ & 0.1844 & 0.3500 & 0.7048 & 0.2197 & 0.4012 & 0.7023 \\
$Z_{23}$ & 0.1187 & 0.2364 & 0.7136 & 0.0692 & 0.1319 & 0.7052 \\
$Z_{31}$ & -0.0071 & -0.0175 & 0.7075 & 0.0032 & 0.0062 & 0.7056 \\
$Z_{32}$ & 0.0845 & 0.1753 & 0.7097 & 0.0996 & 0.2025 & 0.7183 \\
$Z_{33}$ & 0.2403 & 0.4425 & 0.6866 & 0.1553 & 0.2877 & 0.7027
\end{tabular}

Hilbert Local Correlation Coefficients

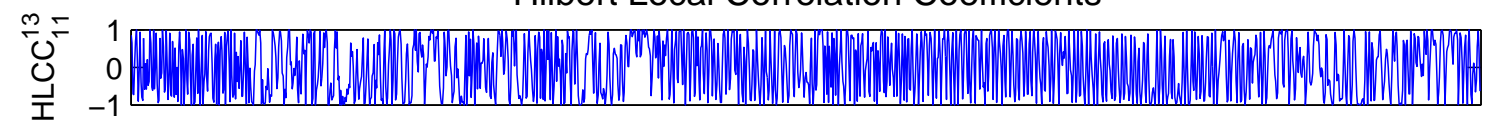

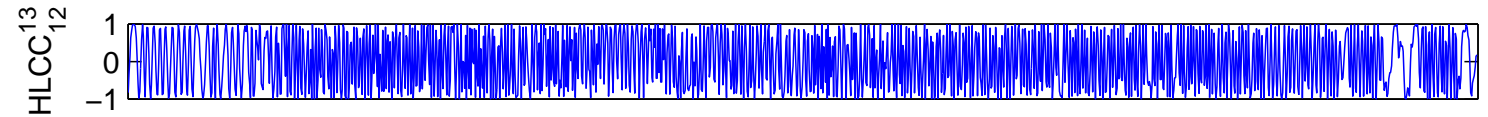

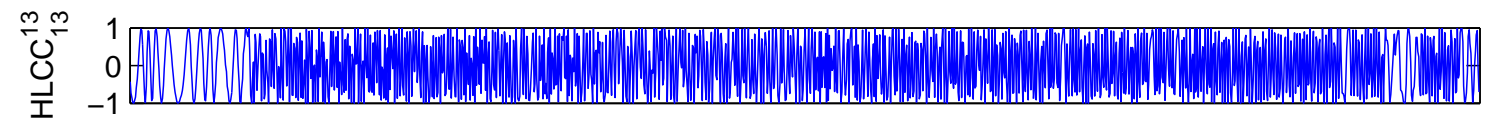

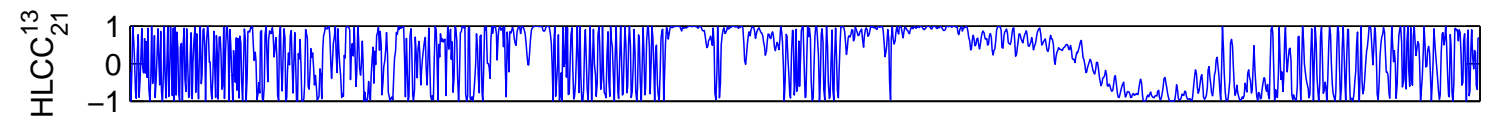

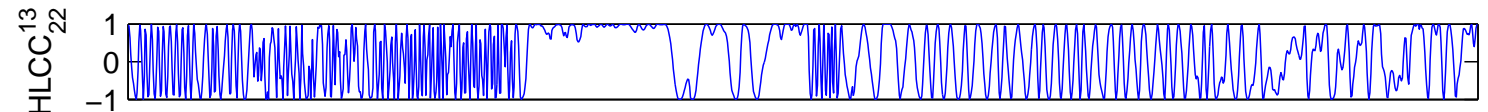

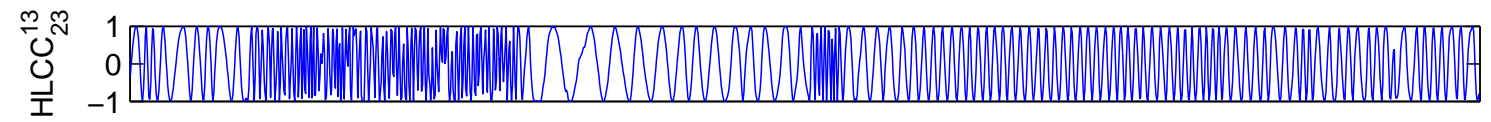
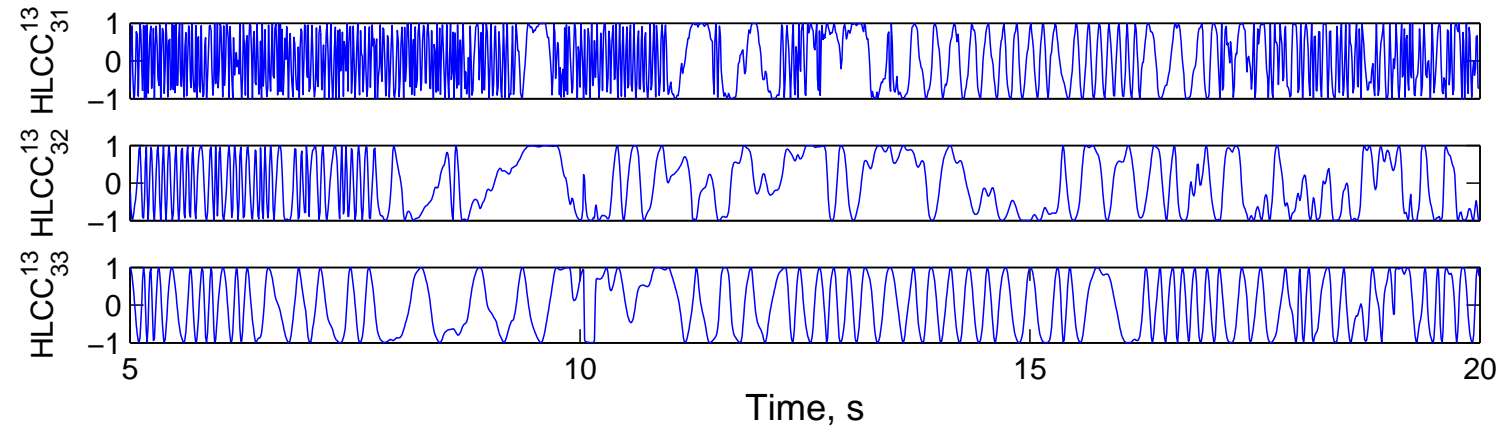

Figure 8. $H L C C_{x y}^{13}$ functions between IMFs of Accel\#1 (top right, Fig. 7) and Accel\#3 (top left, Fig. 7). 


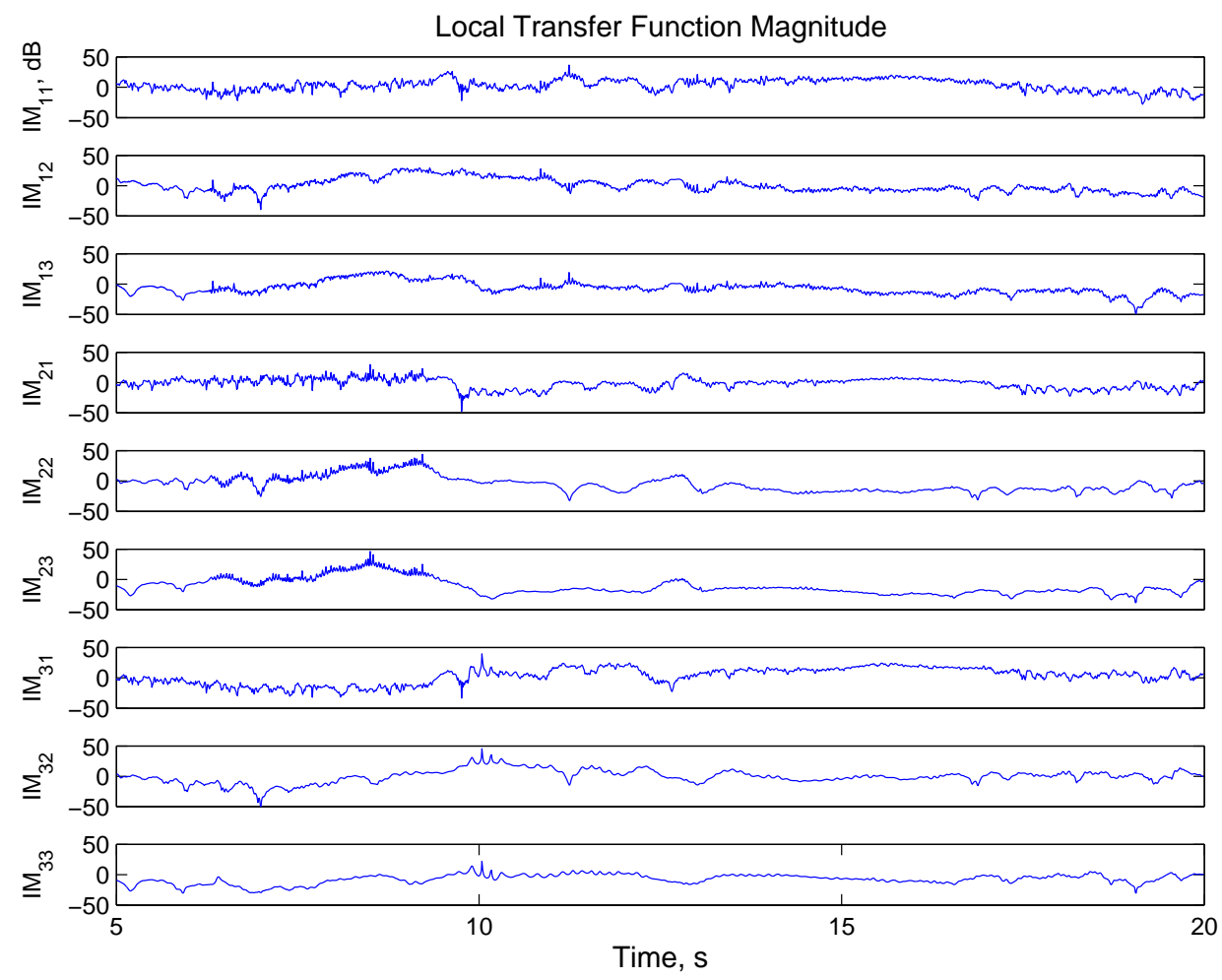

Local Transfer Function Phase

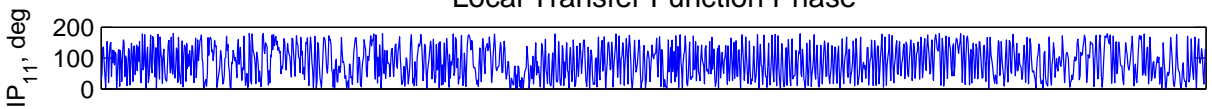

$\cong \begin{gathered}200 \\ \simeq \\ 0\end{gathered}$

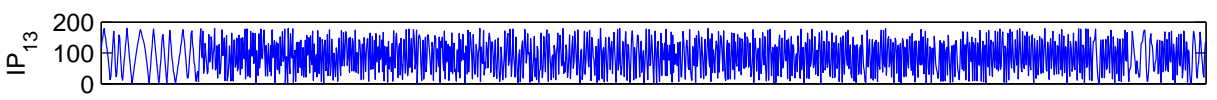

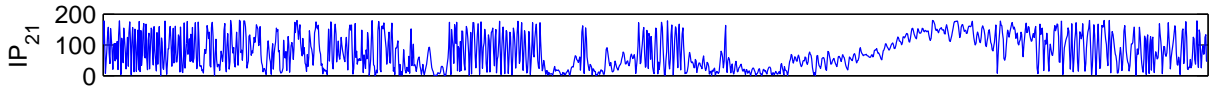

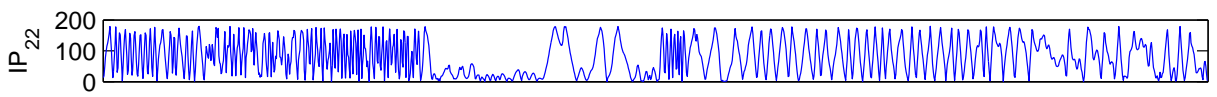

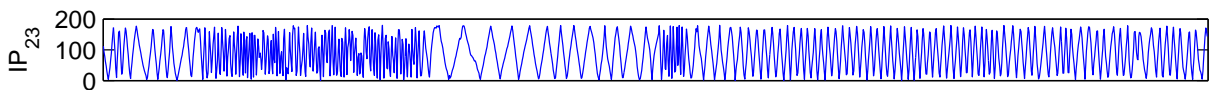

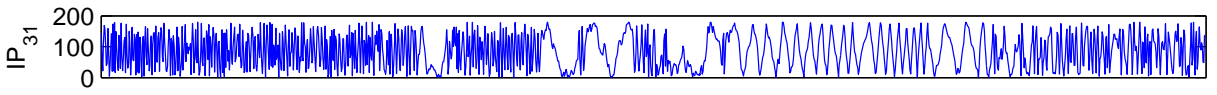

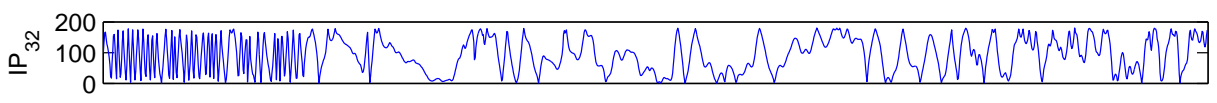

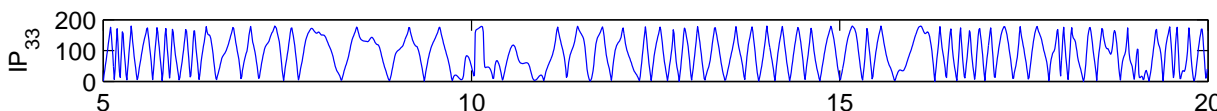

Time, $s$

Figure 9. Instantaneous log-magnitude $\left(I M_{x y}^{a 1}\right.$, top plot set) and phase ( $I P_{x y}^{a 1}$, bottom plot set) of IMF transfer functions from F/A-18 AAW aileron input (Fig. 6) to Accel\#1 (top right, Fig. 7).

13 of 29 


\section{Empirigrams and Empirical Local Correlation Coefficient}

Given two EMDs from different signals, select a common number of IMFs to use for correlation (as three were selected in earlier examples for input and outputs). This is generally not difficult, especially for aeroelasticity data, since the higher-numbered IMFs approach a residual characteristic quickly. From the transformed IMFs, $\left\{Z_{x}(t), Z_{y}(t)\right\}$, define the corresponding set of two-dimensional Hilbert empirigrams, $\left\{H_{x}\left(\eta_{x}, t\right), H_{y}\left(\eta_{y}, t\right)\right\}$, where for each common $\eta=\eta_{x}=\eta_{y}$, the IMF number from the input EMD corresponds with the output EMD. Define in Eq. 6 the Hilbert cross-empirigram

$$
H_{x y}(\eta, t)=H_{x}^{*}(\eta, t) H_{y}(\eta, t)
$$

which correlates the respective IMFs from the two Hilbert empirigrams. Empirigrams relate to time-scale wavelet scalograms, ${ }^{5}$ which relate to time-frequency maps, since scales relate to frequencies in standard wavelet decompositions. Because of the bandpass nature of IMFs discussed previously, a similar construction emanates with the HHT. As with wavelet scalograms, the real part of the cross-empirigram (co-empirigram) gives the instantaneous contribution of each IMF to the correlation between two signals. An Empirical Local Correlation Coefficient, $E L C C(\eta, t)$, is then defined as

$$
\operatorname{ELCC}(\eta, t)=\frac{\operatorname{Re}\left[H_{x y}(\eta, t)\right]}{\left|H_{x y}(\eta, t)\right|}=\frac{\operatorname{Re}\left[H_{x y}(\eta, t)\right]}{\left|H_{x}(\eta, t)\right|\left|H_{y}(\eta, t)\right|}
$$

where $E L C C(\eta, t)$ (between \pm 1 , as the $H L C C$ ) gives the instantaneous contribution between corresponding IMFs from the two signals to the correlation coefficient. Figure 10 shows an imaged decomposition plot of the $E L C C$ between the input signal from Fig. 6 to Accel\#1 in Fig. 7, but includes all nine IMFs from the EMDs of the input and output. The first row represents the contributions of the first input IMF to first output IMF, etc., up to the ninth IMF. In each IMF row there is much oscillation (higher frequency in the first and lower frequency to the ninth) of contributions from corresponding IMFs to the correlation. There are generally stronger correlations over longer time spans in the higher-numbered IMFs (lower frequencies), but the lower-numbered IMF correlations are less obvious due to the higher frequency content. There is a tendency to cycle from high-to-low-to-high [strong(positive)-to-noneto-strong(negative)] correlation very rapidly. A more detailed depiction in the zoomed-in bottom plot, between 10-11 $\mathrm{s}$, demonstrates a rich interplay between correlation of mid-to-lower IMFs (higher frequencies) over the shorter time period. Yet another view is presented in Fig. 11, where the contours are split up discretely in three dimensions showing the heavy emphasis in the higher frequencies because the contours are more congested in each of the IMF levels. These representations highlight the areas of commonality and incongruity between corresponding input-output IMFs.

From the Hilbert spectrum $H(\omega, t)$ the energy spectrum $H^{2}(\omega, t)$ gives instantaneous energy,

$$
I E(t)=\int_{\omega} H^{2}(\omega, t) d \omega
$$

which is an indication of the energy fluctuations with time being weighted by the Hilbert spectrum localized energy over the entire set of IMFs. Corresponding to the signals in Fig. 7 are the instantaneous energy profiles of the output accelerometer responses in Fig. 12 (top set of four plots). The aft wingtip accels (bottom plots) are very similar (from the symmetric aileron input), indicating energy at $\{7-10,13-20,30\}$ s time locations. In this case, with the particular multisine input from Fig. 6 programmed over the $3-35 \mathrm{~Hz}$ frequency range, these times correspond closely to the primary F/A-18 AAW modal frequencies in a corresponding frequency range, i.e., $\{6-9,12-20,30-35\} \mathrm{Hz}$, as will be shown with marginal Hilbert spectra. The forward accelerometer responses in the top plots are also very similar indicating modes near $\{6,12-17,25-32\} \mathrm{Hz}$. Instantaneous energy over a sensor suite is therefore a nonstationary indicator of time-varying energy distribution amongst the sensor array.

\section{Marginal Spectra}

From the Hilbert spectrum $H(\omega, t)$, the marginal spectrum is also defined.

$$
h(\omega)=\int_{t} H(\omega, t) d t
$$

14 of 29 

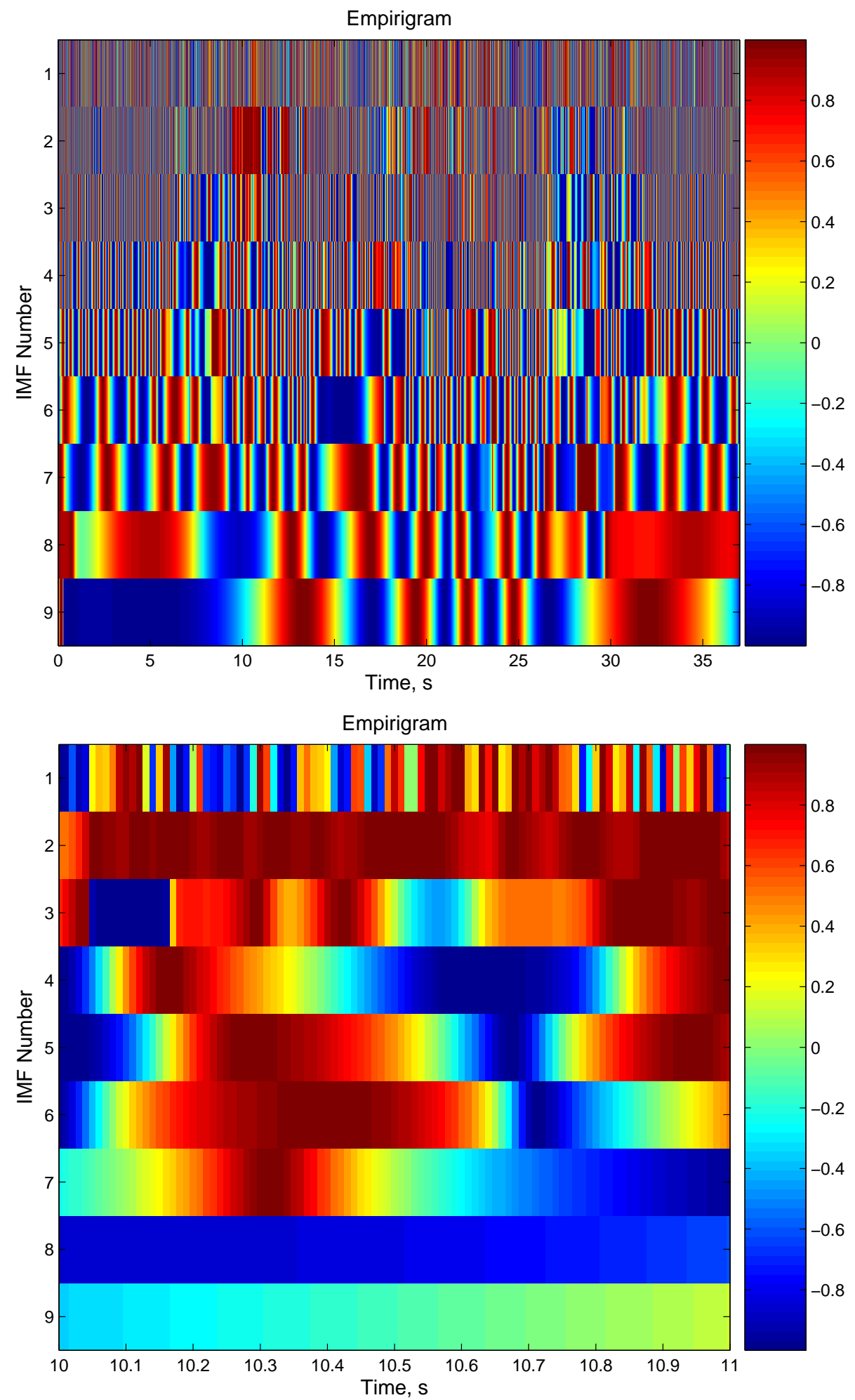

Figure 10. Empirical local correlation coefficient $E L C C$ plots using two different representations of the $E L C C$ between the input signal from Fig. 6 to Accel\#1 in Fig. 7. Top plot is an intrinsic mode component-by-component depiction, starting from the first at the top to the ninth at the bottom, where individual intrinsic functions are clearly delineated. Bottom plot is zoomed-in portion between 10-11 s of the same $E L C C .15$ of 29 


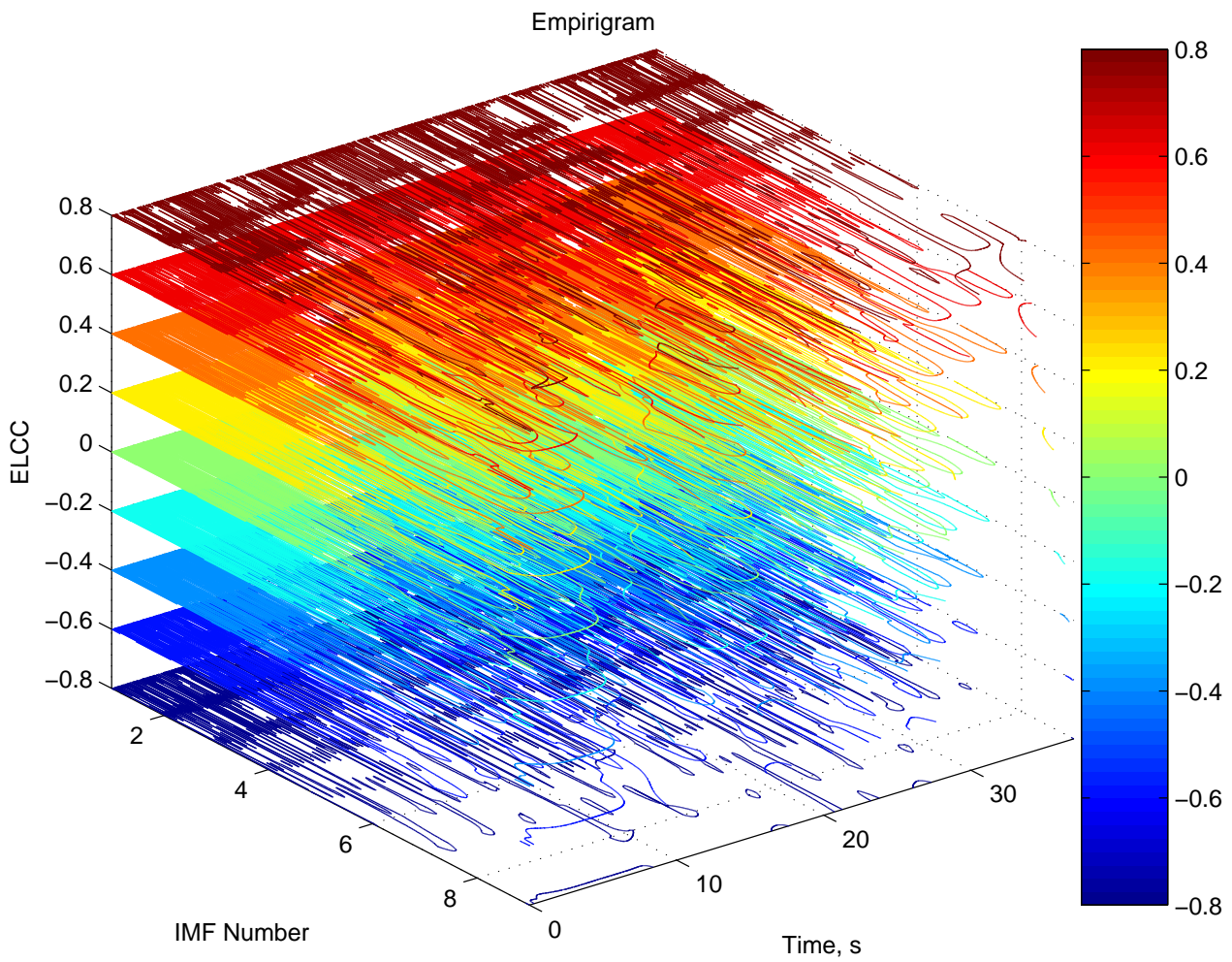

Figure 11. $E L C C$ represented using a three-dimensional contour of the result in Fig. 10. 

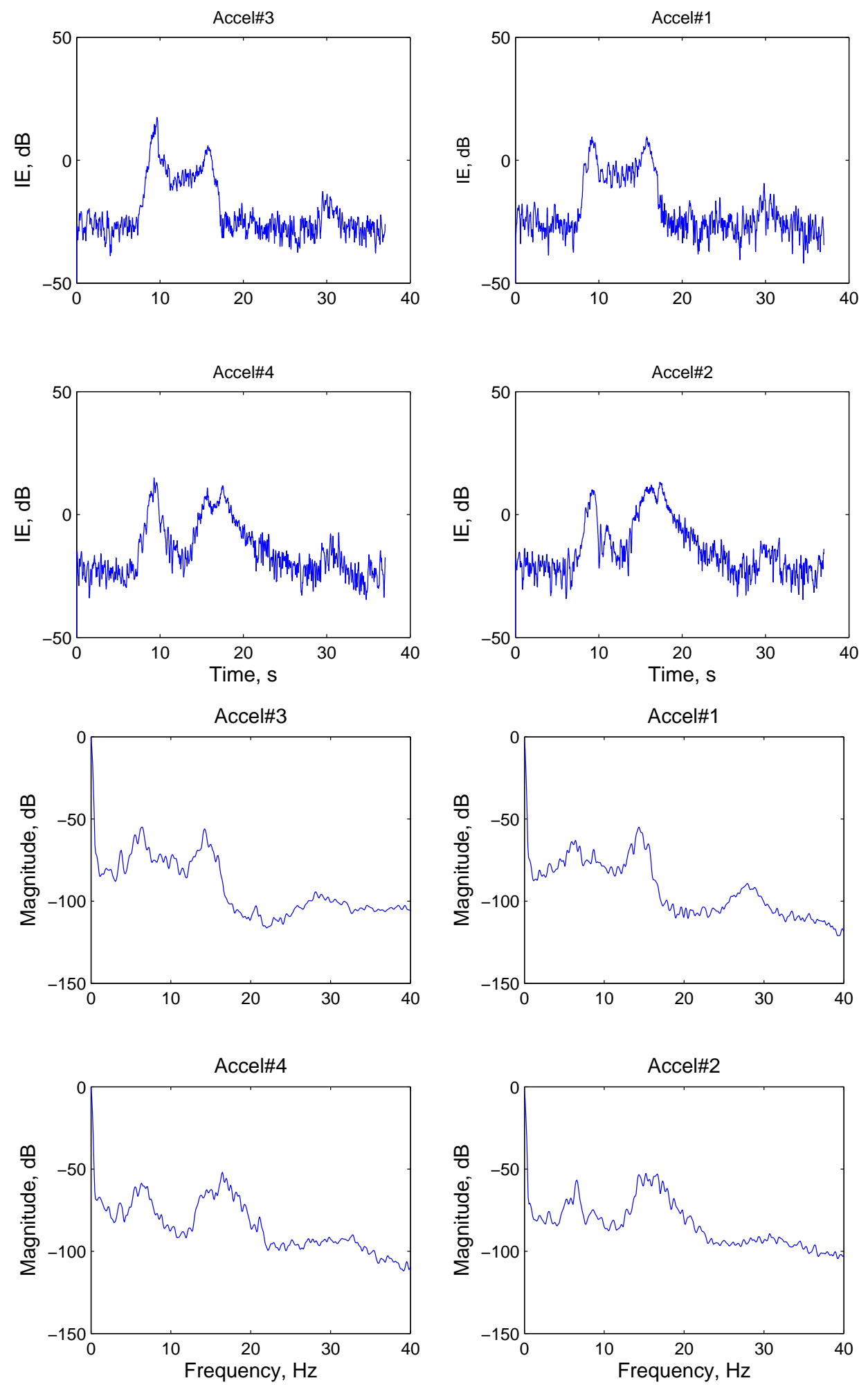

Figure 12. Instantaneous energy (top plot set) and marginal spectra (bottom plot set) of right-fwd wingtip accel (top right, Accel\#1), right-aft wingtip accel (bottom right, Accel\#2), left-fwd wingtip accel (top left, Accel\#3), and left-aft wingtip accel (bottom left, Accel\#4) responses, respectively in each set, from the multisine symmetric aileron command shown in Fig. 6. 
Corresponding to the signals in Fig. 7 are the marginal spectra of the output accelerometer responses in Fig. 12 (bottom set of four plots). The aft wingtip accels (bottom plots) are again similar showing modal response at $\{5-10,12-$ $20,30-35\} \mathrm{Hz}$. The forward accelerometer responses in the top plots are also again very similar indicating modes near $\{6,12-17,25-32\} \mathrm{Hz}$.

The marginal spectrum measures total amplitude (or energy) contributions from each frequency value over the entire data record in a probabilistic sense. Frequency in either $H(\omega, t)$ or $h(\omega)$ is very different from Fourier spectral analysis. In the Fourier analysis the existence of energy at a frequency implies a wave component persisting through the data. Energy at the marginal Hilbert frequency, however, implies a higher likelihood (expected value over time) for such a wave to have appeared locally. As stated earlier, the Hilbert transform of an IMF gives the best fit with a sinusoidal function to the data weighted by $1 / t$, which makes it instantaneous. In fact, the Hilbert spectrum is a weighted nonnormalized joint amplitude-frequency-time distribution in which the weight assigned to each timefrequency cell is the local amplitude. Consequently, frequency in the marginal spectrum indicates only the likelihood that an oscillation with such a frequency exists. Exact occurrence of frequency content is given in the full Hilbert spectrum. The Fourier spectrum is meaningless for nonstationary data, and there is little similarity between Hilbert and Fourier from previous studies ${ }^{8,9}$ for nonstationary data. Also, marginal cross-spectra between signals does not make sense since the time-dependence (causality) is lost and the frequencies are time-ignorant, so an input-output correlation at a certain frequency is meaningless.

The time-frequency Fourier spectrum, a spectrogram, suffers from the same restrictions over time due to windowing distortions. There is a lower bound on the local time-frequency resolution uncertainty product of the spectrogram due to the windowing operation. This limitation is an inherent property of the spectrogram and is not a property of the signal or a fundamental limit. ${ }^{17}$ For many other time-frequency distributions, the local uncertainty product is less than that of the Fourier spectrogram and can be arbitrarily small. These results are contrary to the common notion that the uncertainty principle limits local quantities. Similar considerations apply to window-based filter bank (wavelet) methods. This limitation is due to the window and not to any inherent property of the signal.

This is a key point for application and understanding of HHT analysis, that windowing is not a factor, so standard time-frequency resolution limitations based on the uncertainty principle do not apply. As seen in the on-line analysis, an adaptive sliding window method has good performance simply requiring adequate data in the window to initiate a sifting process for satisfying the two IMF properties for analyticity.

\section{Wing Dynamics Analysis}

Dynamics between different sensors from the same input will now be investigated. This information will be used to guide the analysis of a group of wing accelerometer responses from a single input in terms of input-output correlation contribution for the F/A-18 AAW aircraft ${ }^{18}$ and Aerostructures Test Wing (ATW). ${ }^{12}$

\section{F/A-18 Active Aeroelastic Wing (AAW) Aircraft}

Collective F/A-18 AAW aileron position, used as the multisine input, was obtained as the average of four position transducer measurements from the right and left ailerons. Outputs are twelve wing structural accelerometers located at the left (six accels) and correponding right (six accels) outer wings, all sampled at 400 sps, as designated in Table 2. EMDs were calculated for the input and all outputs, with mean frequencies calculated for all the output IMFs compared to the single input IMFs. Table 3 is a summary of the results. Included are the first six IMFs averaged amongst the accels, percentage differences of these compared to the input IMFs, and standard deviations of output sensor IMFs. The accels correspond very well amongst each other in IMF frequencies (as frequency decreases, from left to right). The comparison to the input frequency is excellent even though input and output EMDs are performed independently. Orthogonality of the IMFs in each case was also very good. This shows that for this type of data, where responses are all from a common input (whether it is known or not), IMFs can be used for sensor-to-sensor correlation and input-output analysis.

Now the concept of the using the essentially-orthogonal analytic IMFs from the inputs and outputs is pursued to establish a multi-loop connotation of input IMFs to output IMFs for correlation and even stability properties. The

18 of 29 
Table 2. F/A-18 AAW wing accelerometer nomenclature.

\begin{tabular}{c|c}
$\begin{array}{c}\text { 3. L-wt-fwd } \\
\text { 3. Left-wingtip forward }\end{array}$ & $\begin{array}{c}\text { 1. R-wt-fwd } \\
\text { 1. Right-wingtip forward }\end{array}$ \\
\hline $\begin{array}{c}\text { 7. L-wof-fwd } \\
\text { 7. Left-wing outer-fold forward }\end{array}$ & $\begin{array}{c}\text { 5. R-wof-fwd } \\
\text { 5. Right-wing outer-fold forward }\end{array}$ \\
\hline $\begin{array}{c}\text { 11. L-wif-fwd } \\
\text { 11. Left-wing inner-fold forward }\end{array}$ & $\begin{array}{c}\text { 9. Right-wing inner-fold forward } \\
\text { 4. L-wt-aft } \\
\text { 4. Left-wingtip aft }\end{array}$ \\
\hline $\begin{array}{c}\text { 8. L-wof-aft } \\
\text { 2. R-wt-aft }\end{array}$ \\
$\begin{array}{c}\text { 8. Left-wing outer-fold aft } \\
\text { 12. L-wif-aft }\end{array}$ & $\begin{array}{c}\text { 6. Right-wing outer-fold aft } \\
\text { 10. R-wif-aft }\end{array}$ \\
12. Left-wing inner-fold aft & 10. Right-wing inner-fold aft
\end{tabular}

Table 3. IMF frequencies from F/A-18 AAW input collective aileron position and outer wing response data.

\begin{tabular}{l|ccc|ccc} 
Signal & IMF1 & IMF2 & IMF3 & IMF4 & IMF5 & IMF6 \\
\hline 0. Input & 116.62 & 59.61 & 29.34 & 16.75 & 9.05 & 4.90 \\
\hline 1. R-wt-fwd & 99.05 & 56.94 & 27.90 & 12.56 & 6.96 & 3.62 \\
2. R-wt-aft & 123.85 & 71.36 & 35.15 & 16.83 & 9.35 & 5.02 \\
3. L-wt-fwd & 114.47 & 66.05 & 33.78 & 15.06 & 7.95 & 5.32 \\
4. L-wt-aft & 127.56 & 78.71 & 41.73 & 21.06 & 11.92 & 6.58 \\
5. R-wof-fwd & 105.83 & 58.52 & 32.06 & 17.17 & 9.34 & 5.47 \\
6. R-wof-aft & 96.31 & 54.96 & 29.24 & 14.91 & 8.64 & 4.63 \\
7. L-wof-fwd & 115.93 & 58.77 & 30.88 & 16.59 & 8.66 & 4.32 \\
8. L-wof-aft & 102.99 & 52.76 & 27.14 & 15.35 & 8.65 & 4.83 \\
9. R-wif-fwd & 112.19 & 60.61 & 31.99 & 17.78 & 9.90 & 5.17 \\
10. R-wif-aft & 115.39 & 64.07 & 36.09 & 19.05 & 10.29 & 5.72 \\
11. L-wif-fwd & 124.91 & 66.06 & 32.99 & 18.32 & 10.53 & 5.19 \\
12. L-wif-aft & 129.41 & 66.05 & 34.02 & 17.97 & 9.66 & 4.92 \\
\multicolumn{7}{|c}{ Average Output IMF Frequencies } \\
\hline Absolute & 114.0 & 62.9 & 32.8 & 16.9 & 9.3 & 5.1 \\
Wrt Input & $2.3 \%$ & $5.2 \%$ & $10.4 \%$ & $0.8 \%$ & $2.9 \%$ & $3.2 \%$ \\
\multicolumn{7}{c}{ Output IMF Frequency STDs } \\
\hline Absolute & 11.2 & 7.3 & 4.0 & 2.2 & 1.3 & 0.7 \\
Wrt Mean & $9.8 \%$ & $11.7 \%$ & $12.1 \%$ & $13.2 \%$ & $13.9 \%$ & $14.4 \%$
\end{tabular}

input IMFs are interpreted as an orthogonal decomposition of the input(s), and the same for output IMFs for output(s). This can be generalized to multi-input-multi-output (MIMO) signal analysis where in reality each signal is represented by its EMD. Recall from the transformed input-output IMFs, $\left\{Z_{x}(t), Z_{y}(t)\right\}$, the corresponding set of empirigrams $\left\{H_{x}\left(\eta_{x}, t\right), H_{y}\left(\eta_{y}, t\right)\right\}$ were defined in Eq. 6 for each set of $\eta=\eta_{x}=\eta_{y}$ common IMFs from the 
input and output EMDs. For the analysis described here it is not necessary to use an identical number, $\eta$, of IMFs from input and output (but this restriction is maintained here for simplicity). Then the Hilbert cross-empirigram $H_{x y}(\eta, t)=H_{x}^{*}(\eta, t) H_{y}(\eta, t)$ correlates the respective analytic IMFs from the two Hilbert empirigrams. Now define

$$
\sigma_{x y}(\eta)=\sigma\left[H_{x}^{*}(\eta, t) H_{y}^{T}(\eta, t)\right]
$$

where $\sigma_{x y}$ represents the $\eta$-vector of singular values from the singular value decomposition (SVD) of the product of the empirigrams. The singular values represent relative contributions from the principal cross-correlation analytic IMFs as a result of correlation of all input analytic IMFs to all output analytic IMFs over the entire time span. Therefore, higher $\sigma$-valued cross-analytic IMFs have more input-output significance in terms of operator norm from input to output. The maximum singular value, $\bar{\sigma}_{x y}=\max _{\eta}\left(\sigma_{x y}(\eta)\right)$, of this input-output operator corresponds to the structured singular value with a full-complex uncertainty block structure. In this context, for $M^{\eta \times \eta}=H_{x}^{*}(\eta, t) H_{y}^{T}(\eta, t)$, a complex matrix operator of input analytic IMFs to output analytic IMFs, $\mu_{\Delta}(M)$ is a measure of the smallest uncertainty $\Delta=\mathbf{C}^{\eta \times} \eta$ (note that if $\eta_{x} \neq \eta_{y}$, then $\Delta=\mathbf{C}^{\eta_{\mathbf{y}} \times \eta_{\mathbf{x}}}$ is not square) that causes instability if interpreted as a constant matrix feedback loop, ${ }^{30}$

$$
\mu_{\left\{\Delta=\mathbf{C}^{\eta \times \eta}\right\}}(M)=\bar{\sigma}\left[H_{x}^{*}(\eta, t) H_{y}^{T}(\eta, t)\right]
$$

and for scalar uncertainty structure $\Delta=\left\{\delta I_{\eta}: \delta \in \mathbf{C}\right\}$ representing diagonal structure between input-output IMFs,

$$
\mu_{\Delta}(M)=\rho\left[H_{x}^{*}(\eta, t) H_{y}^{T}(\eta, t)\right]
$$

where $\rho$ is the spectral radius (largest magnitude eigenvalue). This is distinctly different from the full-block structure in that uncertainty is only between corresponding input-output analytic IMFs (similar dominant frequencies), while ignoring uncertainty across different analytic IMFs (of different dominant frequencies) between input and output. This is obviously less conservative but generally less realistic as well, especially for nonlinear effects which cross frequencies. Larger $\mu_{\Delta}$ values in either case represent effects of uncertainty between input and output such that higher correlated IMFs relate to more sensitivity to uncertainty at those dominant IMF frequencies.

For MIMO signal analysis, it would be most appropriate to combine complex blocks (either full or scalar) for each input-output into a multi-block structure, where each complex sub-block corresponds to an input-output analytic IMF complex uncertainty structure. In computation this is often expanded in a block-diagonal context with repeated scalar and full blocks, ${ }^{30}$ where in this case each of these blocks would correspond to a single input-output analytic IMF uncertainty structure.

Table 4. Normalized $\mu_{\Delta}$ results from F/A-18 AAW left and right wing acceleration data.

\begin{tabular}{l|ccc|ccc} 
Full & 3. L-wt-fwd & 7. L-wof-fwd & 11. L-wif-fwd & 9. R-wif-fwd & 5. R-wof-fwd & 1. R-wt-fwd \\
Block & 4. L-wt-aft & 8. L-wof-aft & 12. L-wif-aft & 10. R-wif-aft & 6. R-wof-aft & 2. R-wt-aft \\
\hline Fwd-wing & 0.29 & 0.29 & 0.73 & 1.00 & 0.62 & 0.80 \\
Aft-wing & 0.65 & 0.67 & 0.56 & 0.19 & 0.31 & 0.72 \\
\hline \hline Scalar & 3. L-wt-fwd & 7. L-wof-fwd & 11. L-wif-fwd & 9. R-wif-fwd & 5. R-wof-fwd & 1. R-wt-fwd \\
Block & 4. L-wt-aft & 8. L-wof-aft & 12. L-wif-aft & 10. R-wif-aft & 6. R-wof-aft & 2. R-wt-aft \\
\hline Fwd-wing & 0.11 & 0.13 & 0.19 & 1.00 & 0.47 & 0.49 \\
Aft-wing & 0.23 & 0.42 & 0.27 & 0.11 & 0.06 & 0.34
\end{tabular}

To compare input-to-output correlations from aileron position to wing accels for different uncertainty structures, structured singular values were computed for the full-block and scalar-block structures between the collective aileron position and F/A-18 AAW wing accelerometer responses, then normalized with respect to the largest of the group. Table 4 lists the results for both types of uncertainty structures. Accelerometer\#9 has the highest correlation $(=1.00)$ with the input aileron position in either case. Interestingly, comparison between left and right wing accels is poor 

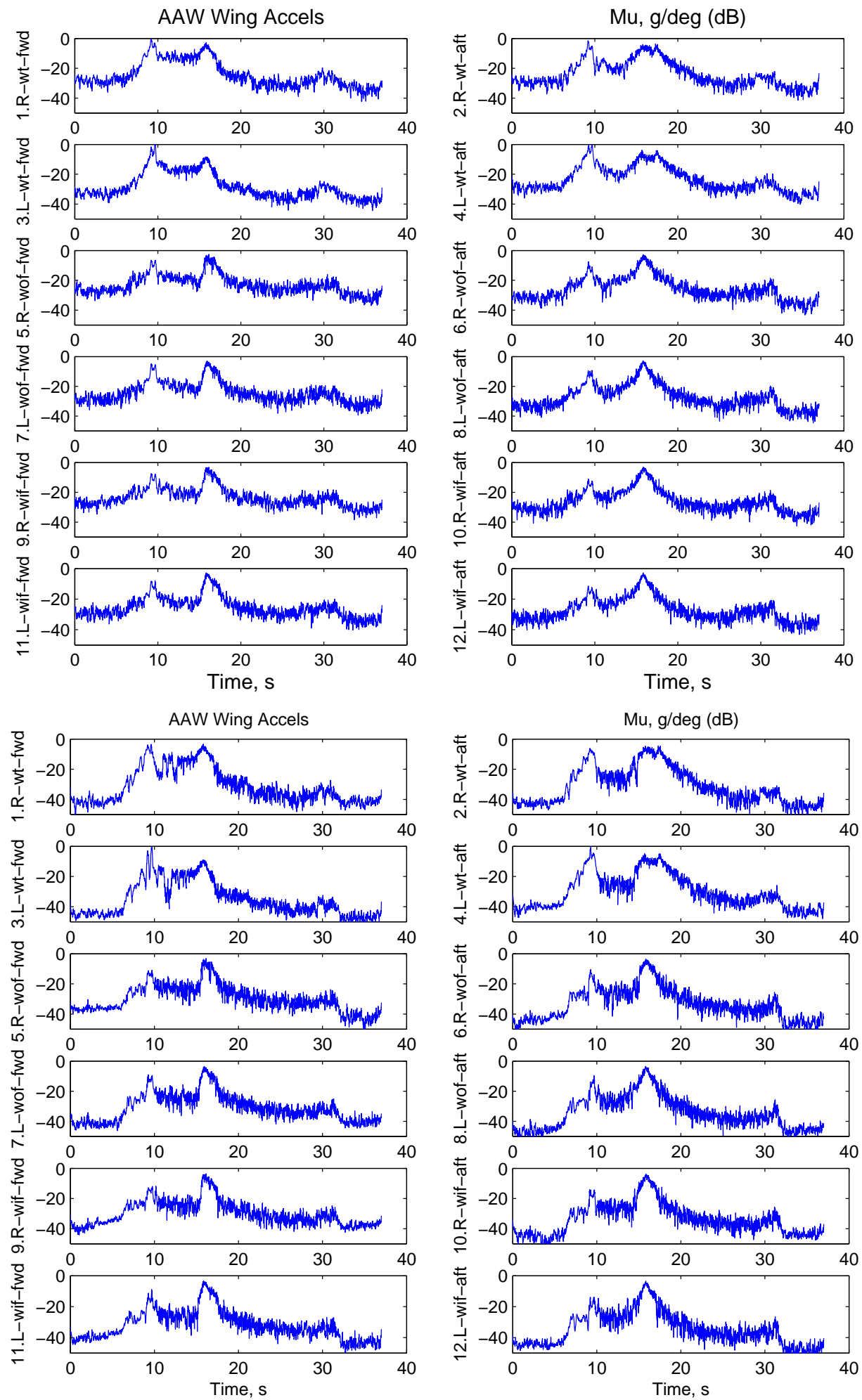

Figure 13. Time-varying $\mu_{\Delta}(M, t)$ (dB) for full-block (top plots) and scalar-block (bottom plots) uncertainty. 
even from a symmetric aileron input, thereby indicating asymmetry and/or nonlinearity. Lower $\mu_{\Delta}$ values indicate a degree of robustness to uncertainty in a feedback stability sense, and as pointed out previously, scalar uncertainty is prevalently less conservative (as evidenced from absolute values before normalizing since the normalization factor is almost equal between the uncertainty structures). If an apriori bound on gain levels (normalization) between inputoutput is defined such that $\|\Delta\|_{\infty}<1$ is considered acceptable in some sense (stability, health diagnostic, safety margin), then $\mu_{\Delta}$ has an absolute (scaled) interpretation such that $\mu_{\Delta}>1$ indicates that an acceptable threshold has been exceeded, and this can be used as a health, stability, or safety monitor.

A time-dependent interpretation is also available by calculating $\mu_{\Delta}(M, t)$ at each time point.

$$
\begin{array}{rll}
\mu_{\Delta}(M, t) & =\rho\left[H_{x}^{*}\left(\eta, t_{i}\right) H_{y}^{T}\left(\eta, t_{i}\right)\right] \forall t_{i} & \text { (scalar-block uncertainty structure) } \\
& =\bar{\sigma}\left[H_{x}^{*}\left(\eta, t_{i}\right) H_{y}^{T}\left(\eta, t_{i}\right)\right] \forall t_{i} & \text { (full-block uncertainty structure) }
\end{array}
$$

In Fig. 13 are plots of $\mu_{\Delta}(M, t)$ for each input-to-output (aileron-to-accel) normalized analytic IMFs where it is evident that values close to one $(0 \mathrm{~dB})$ are near a unity operator norm limit. Again, this depends on the bound $\|\Delta\|_{\infty}<1$ indicating an acceptable threshold, in this case maximum input-to-output gain normalized to one. Values close to one $(0 \mathrm{~dB})$ approach the acceptable limit. This uncertainty structure application has implications for model validation in the time domain. ${ }^{19}$

\section{Aerostructures Test Wing (ATW)}

Another example which includes an actual instability is taken from Aerostructures Test Wing ${ }^{12}$ (ATW) flight test data. The input is a sine sweep PZT voltage and outputs are three wingtip accelerometer responses. The EMDs of the PZT input and center wingtip accelerometer output near the flutter condition are displayed in Fig. 14. Flutter response
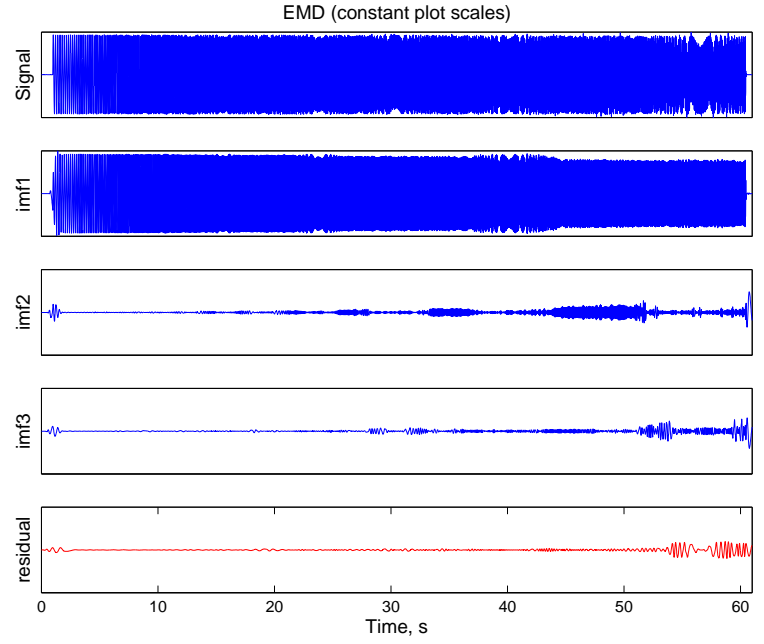

Figure 14. Empirical mode decomposition of the ATW input PZT (left plot) and center wingtip accelerometer (right plot) at Mach 0.82, 10,000 feet $(3,048 \mathrm{~m})$ altitude, just before flutter (original signal at top and residual at bottom in each set).

EMDs of the center wingtip accelerometer and corresponding $\mu_{\Delta}(M, t)$ plots between input analytic IMFs and all three wingtip accelerometer output analytic IMFs are shown in Fig. 15. The input PZT was not activated during the flutter occurrence, so the input is essentially a small arbitrary oscillation about zero. It is evident that at the point of instability past $7.5 \mathrm{~s}, \mu_{\Delta}(M, t)$ appropriately approaches a value of one $(0 \mathrm{~dB})$ since the input and output are normalized to unity, and assuming this limit corresponds to the limit of stability, and output response approaches its upper limit at flutter. 

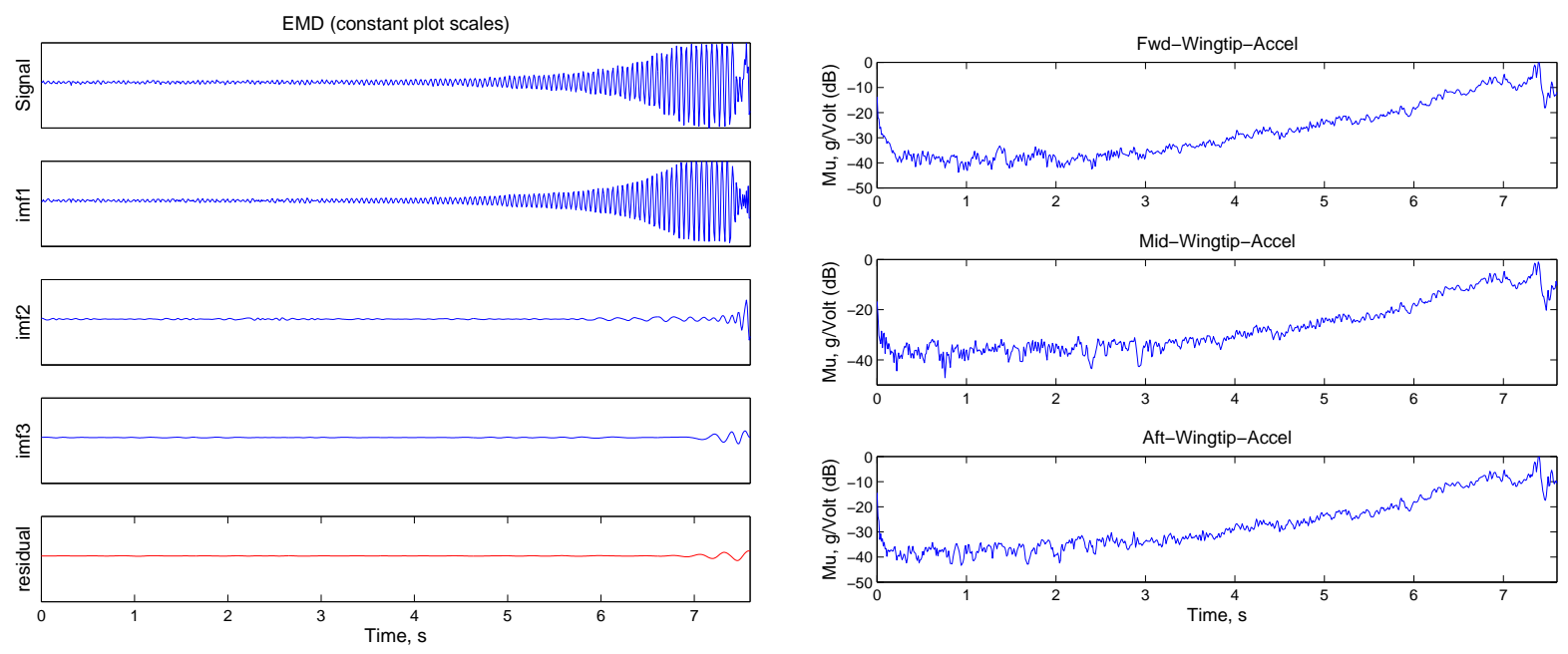

Figure 15. Empirical mode decomposition of the center wingtip accelerometer (left plot) near Mach $0.83,10,000$ feet $(3,048$ m) altitude, at flutter (original signal at top and residual at bottom), and time-varying $\mu_{\Delta}(M, t)$ for full-block uncertainty between input and output IMFs of the three wingtip accelerometer outputs (right plots).

\section{Parameter Estimation Using the Hilbert-Huang Transform}

The Hilbert transform can used for estimating modal parameters such as natural frequencies and damping ratios. For a single-mode system, the damped natural frequency can be easily determined from the Hilbert transform of the impulse response function. In this case, the damped natural frequency $\omega_{d}$ is given by the slope of the instantaneous phase angle plotted as a function of time, and damping ratio $\zeta$ is estimated in a straightforward manner. ${ }^{23}$

For a linear single-mode system characterized by a pair of complex conjugate eigenvalues, the eigenvalues are directly related to the modal parameters as $-\zeta \omega_{n}+\omega_{d}$, where $\omega_{n}$ denotes the natural frequency. The impulse response function is given by

$$
h(t)=\frac{1}{m \omega_{d}} \mathrm{e}^{-\zeta \omega_{n} t} \sin \left(\omega_{d} t\right)
$$

where $m$ denotes the mass. Provided the damping ratio is small, the following relationships hold ${ }^{23}$

$$
\begin{aligned}
\mathrm{H}\left\{\mathrm{e}^{-\zeta \omega_{n} t} \sin \left(\omega_{d} t\right)\right\} & =\mathrm{e}^{-\zeta \omega_{n} t} \cos \left(\omega_{d} t\right) \\
\mathrm{H}\left\{\mathrm{e}^{-\zeta \omega_{n} t} \cos \left(\omega_{d} t\right)\right\} & =-\mathrm{e}^{-\zeta \omega_{n} t} \sin \left(\omega_{d} t\right)
\end{aligned}
$$

where again $\mathrm{H}$ denotes the Hilbert transform. Therefore, the Hilbert transform of the impulse response function can be written as

$$
\mathrm{H}\{h(t)\}:=\tilde{h}(t)=\frac{1}{m \omega_{d}} \mathrm{e}^{-\zeta \omega_{n} t} \cos \left(\omega_{d} t\right) .
$$

The impulse response function can then be viewed as the real part of the following analytic signal ${ }^{23}$

$$
\begin{aligned}
z(t) & =h(t)+\mathrm{i} \tilde{h}(t) \\
& =\frac{1}{m \omega_{d}} \mathrm{e}^{-\zeta \omega_{n} t} \sin \left(\omega_{d} t\right)+\mathrm{i} \frac{1}{m \omega_{d}} \mathrm{e}^{-\zeta \omega_{n} t} \cos \left(\omega_{d} t\right) .
\end{aligned}
$$

The amplitude of this signal is given by

$$
a(t)=\sqrt{h(t)^{2}+\tilde{h}(t)^{2}}=\frac{1}{m \omega_{d}} \mathrm{e}^{-\zeta \omega_{n} t}
$$


which represents the envelope of the impulse response. Taking the natural logarithm of the amplitude yields

$$
\ln (a(t))=-\zeta \omega_{n} t-\ln \left(m \omega_{d}\right) .
$$

The slope $\sigma$ of the natural $\log$ of $a(t)$ plotted against time gives $\sigma=-\zeta \omega_{n}$. Given that $\omega_{d}$ can be obtained from the slope of the phase plotted against time, and using the definition of the damped natural frequency, there results $\omega_{n}$,

$$
\begin{aligned}
\omega_{d} & =\sqrt{1-\zeta^{2}} \omega_{n} \\
\omega_{d}^{2} & =\left(1-\zeta^{2}\right) \omega_{n}^{2}=\omega_{n}^{2}-\sigma^{2} \\
\omega_{n} & =\sqrt{\omega_{d}^{2}+\sigma^{2}}
\end{aligned}
$$

and the damping ratio can be calculated as $\zeta=\sigma / \omega_{n}$. This approach to damping estimation is in the same spirit as the logarithmic decrement technique commonly used in structural dynamics.

Most systems have multiple modes, however, which implies that the impulse response function will generally contain contributions from several modes. Therefore, because they are valid only for single-mode systems, logarithmic decrement or the approach based on the Hilbert transform cannot be used for estimating damping. As emphasized earlier, the Hilbert transform does not yield meaningful frequency information for multiple-mode systems since it attempts to identify a single instantaneous frequency at each time step. The Hilbert-Huang algorithm makes it possible to apply the above technique for damping and frequency estimation because it can decompose a multi-component signal into a series of single-component signals through the EMD process. By performing EMD on the impulse response function of a multiple-mode system, the contributions of the different modes can be extracted and analyzed separately. This approach has been applied by Yang et al. ${ }^{26-28}$ in a series of papers in which the Hilbert-Huang algorithm was used for parameter estimation of several multiple degree-of-freedom structures. A similar analysis is used here to estimate damping ratios and frequencies for aeroelastic systems.

As an example, consider a prototypical linear pitch-plunge aeroelastic system that has been studied extensively in the literature. The system consists of an airfoil with pitch and plunge degrees of freedom, and the input is the deflection $\beta$ of the trailing edge flap. The equations of motion for this system are given by

$$
\begin{aligned}
{\left[\begin{array}{cc}
m & m x_{\alpha} b \\
m x_{\alpha} b & I_{\alpha}
\end{array}\right]\left\{\begin{array}{c}
\ddot{h} \\
\ddot{\alpha}
\end{array}\right\}+\left[\begin{array}{cc}
c_{h} & 0 \\
0 & c_{\alpha}
\end{array}\right]\left\{\begin{array}{c}
\dot{h} \\
\dot{\alpha}
\end{array}\right\}+\left[\begin{array}{cc}
k_{h} & 0 \\
0 & k_{\alpha}
\end{array}\right]\left\{\begin{array}{c}
h \\
\alpha
\end{array}\right\} } \\
=\rho U^{2} b\left[\begin{array}{c}
c_{l_{\alpha}}\left(\alpha+\frac{1}{U} \dot{h}+\frac{1}{U}\left(\frac{1}{2}-a\right) b \dot{\alpha}\right) \\
c_{m_{\alpha}}\left(b \alpha+\frac{1}{U} b \dot{h}+\frac{1}{U}\left(\frac{1}{2}-a\right) b^{2} \dot{\alpha}\right)
\end{array}\right]+\rho U^{2} b\left[\begin{array}{c}
-c_{l_{\beta}} \\
c_{m_{\beta}} b
\end{array}\right] \beta
\end{aligned}
$$

where $h$ denotes the plunge displacement of the airfoil and $\alpha$ represents the pitch angle. Left-hand side terms describe the structural dynamics of the system while right-hand side terms represent quasi-steady aerodynamic forces and moments. In this study, the flow velocity $U$ is varied until the linear flutter speed is reached at approximately $U=$ $11.8 \mathrm{~m} / \mathrm{s}$. The values of the other system parameters are held constant as listed in Table 5 .

Table 5. Parameters for the pitch-plunge aeroelastic system.

$$
\begin{array}{rlllll}
m & =12.387 \mathrm{~kg} & a & =-0.6 & c_{l_{\alpha}}=6.28 & k_{h}=2844.4 \mathrm{~N} / \mathrm{m} \\
I_{\alpha} & =0.065 \mathrm{~m}^{2} \mathrm{~kg} & x_{\alpha} & =0.2466 & c_{m_{\alpha}}=-0.628 & k_{\alpha}=2.82 \mathrm{Nm} \\
\rho & =1.225 \mathrm{~kg} / \mathrm{m}^{3} & c_{h}=27.43 \mathrm{~kg} / \mathrm{s} & c_{l_{\beta}}=3.358 & \\
b & =0.135 \mathrm{~m} & c_{\alpha}=0.036 \mathrm{~m}^{2} \mathrm{~kg} / \mathrm{s} & c_{m_{\beta}}=-0.635
\end{array}
$$

Because this system has two outputs, pitch and plunge, there are two impulse response functions that can be measured. Each of these functions generally contains contributions from both modes of the system. As an example, 

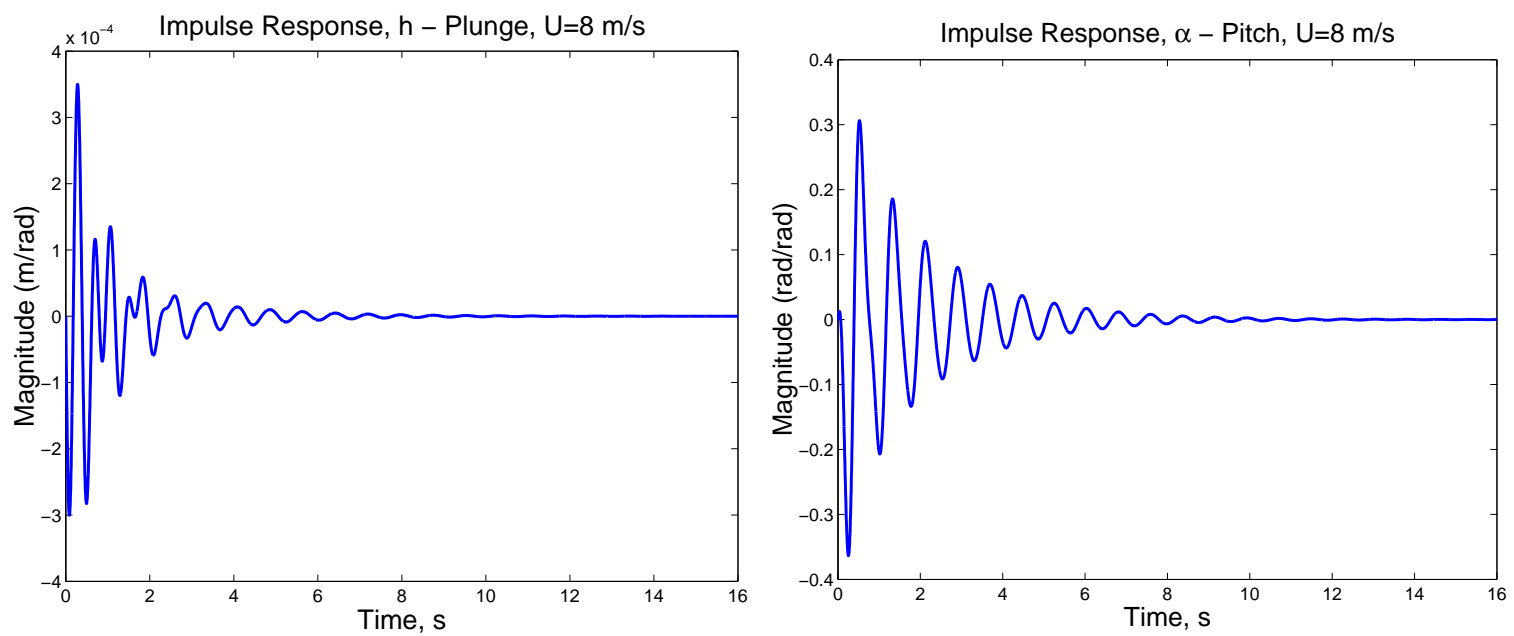

Figure 16. Plunge and pitch impulse response functions for $U=8 \mathbf{m} / \mathbf{s}$
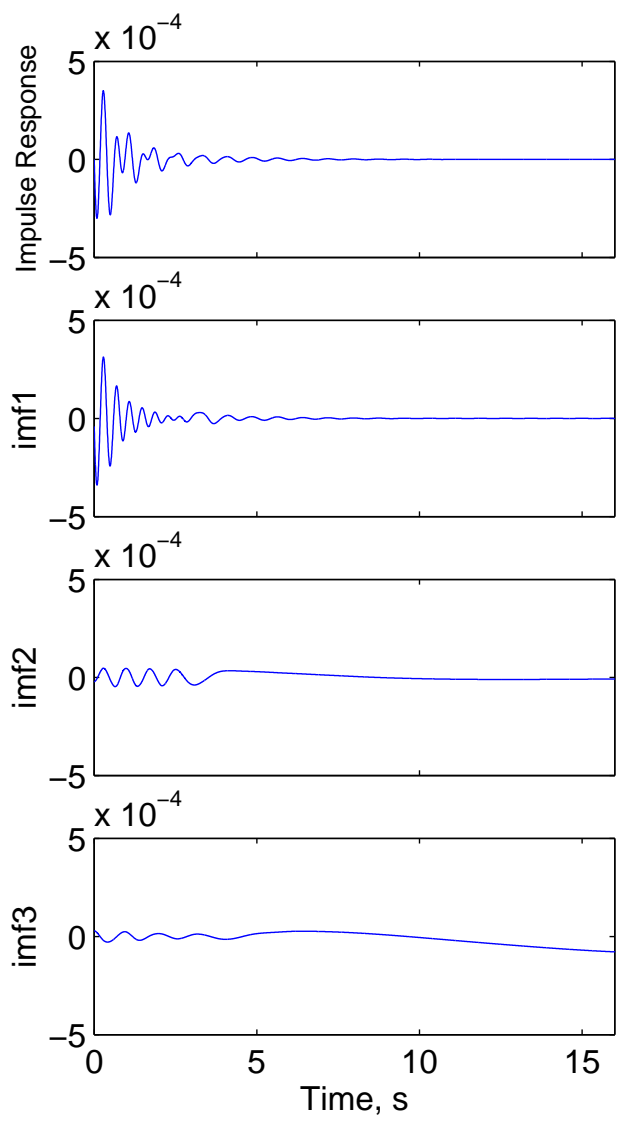

Figure 17. Empirical mode decomposition of the plunge impulse response function $(\mathbf{m} / \mathrm{rad})$ at $U=8 \mathrm{~m} / \mathrm{s}$. 
Fig. 16 depicts the pitch and plunge impulse response functions for a flow velocity of $U=8 \mathrm{~m} / \mathrm{s}$. These were obtained by simulating the pitch and plunge responses to an impulse applied to the trailing edge flap. The plunge impulse responses are used over a range of flow velocities to measure frequencies and damping ratios using the Hilbert-Huang algorithm. These impulse response functions are obtained by way of simulation in this paper, but in practice they can be obtained by way of system identification techniques such as taking the inverse Fourier transform of the measured frequency response function or identifying first-order Volterra kernels from measured input-output data.
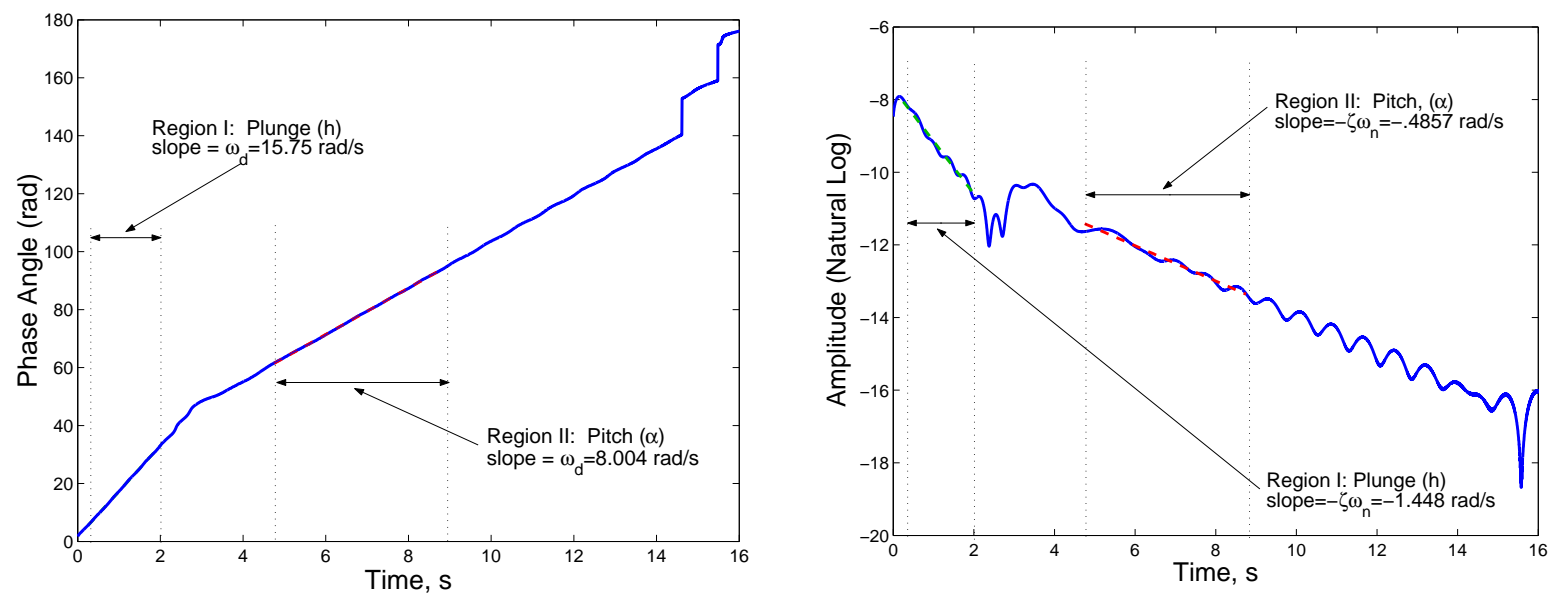

Figure 18. Instantaneous phase and amplitude plots used to estimate modal parameters from the first IMF of the plunge impulse response function at $U=8 \mathrm{~m} / \mathbf{s}$.

The plunge impulse response functions were used to estimate the damped natural frequencies, damping ratios, and natural frequencies over a range of flow velocities. The results are summarized in Table 6, which lists the actual and estimated damped natural frequencies and damping ratios for flow velocities ranging from $U=1 \mathrm{~m} / \mathrm{s}$ to $U=11.9 \mathrm{~m} / \mathrm{s}$ (just past the linear flutter speed). The actual frequencies and damping ratios were calculated by solving for the eigenvalues of the system. The estimation of the modal parameters for $U=8 \mathrm{~m} / \mathrm{s}$ is demonstrated in Figs. 17 and 18. Figure 17 illustrates the EMD of the plunge impulse response function at $U=8 \mathrm{~m} / \mathrm{s}$. In this case, both modes of vibration are evident in the first intrinsic mode function. This is because the first, higher-frequency mode damps out quickly and the second mode persists for a longer period of time. Therefore, the frequencies and damping ratios of both modes were estimated by using different portions of the first IMF, as depicted in Fig. 18. The figure shows plots of the instantaneous phase and the natural log of the amplitude of the first IMF. Recall that the slopes of these lines are used to estimate the modal parameters. The initial portion of the amplitude plot is not a straight line because of initial transients in the IMF and possible boundary effects. Therefore, the portions of the plot that most resembled straight lines were used for the slope calculations for each mode, as labeled in the figure. Because this analysis technique is only approximate depending on the magnitude of the damping ratio (increased error for larger damping), the amplitude curve tends to oscillate about an average slope. Therefore, the slope in each case was obtained using a linear least-squares curve-fit of the data. ${ }^{26-28}$

The results presented in Table 6 show that the estimates of the damped natural frequencies and damping ratios are fairly accurate in most cases. As the linear flutter speed (approximately $U=11.8 \mathrm{~m} / \mathrm{s}$ ) is approached, the plunge mode is not discernible in the IMFs. In this regime, the system behaves essentially as a single-mode system and it was not possible to estimate the damping and frequency of the plunge mode. The actual and estimated damping ratios for each mode are plotted as a function of flow velocity in Fig. 19.

A few observations are now presented. The first IMF was used to estimate the parameters of both modes. The analysis would be somewhat cleaner if each mode appeared in a different IMF. This is indeed the case in the work of Yang et al. ${ }^{26-28}$ in which the frequency range of each IMF is controlled in the EMD process. Therefore, controlling the 
Table 6. Actual and estimated damped frequencies and damping ratios.

\begin{tabular}{|c|c|c|c|c|c|}
\hline Velocity $(\mathrm{m} / \mathrm{s})$ & & Actual $\omega_{d}(\mathrm{~Hz})$ & Estimated $\omega_{d}(\mathrm{~Hz})$ & Actual $\zeta$ & Estimated $\zeta$ \\
\hline \multirow[t]{2}{*}{1} & Plunge & 2.765 & 2.735 & .0854 & .0874 \\
\hline & Pitch & 1.027 & 1.022 & .0441 & .0444 \\
\hline \multirow[t]{2}{*}{3} & Plunge & 2.739 & 2.709 & .0855 & .0881 \\
\hline & Pitch & 1.057 & 1.050 & .0503 & .0490 \\
\hline \multirow[t]{2}{*}{5} & Plunge & 2.684 & 2.655 & .0862 & .0895 \\
\hline & Pitch & 1.119 & 1.096 & .0553 & .0591 \\
\hline \multirow[t]{2}{*}{7} & Plunge & 2.595 & 2.567 & .0877 & .0926 \\
\hline & Pitch & 1.217 & 1.214 & .0590 & .0579 \\
\hline \multirow[t]{2}{*}{9} & Plunge & 2.454 & 2.452 & .0906 & .0957 \\
\hline & Pitch & 1.366 & 1.362 & .0607 & .0637 \\
\hline \multirow[t]{2}{*}{11} & Plunge & 2.198 & - & .1012 & - \\
\hline & Pitch & 1.628 & 1.619 & .0544 & .0544 \\
\hline \multirow[t]{2}{*}{11.5} & Plunge & 2.073 & - & .1174 & - \\
\hline & Pitch & 1.755 & 1.744 & .0392 & .0394 \\
\hline \multirow[t]{2}{*}{11.6} & Plunge & 2.040 & - & .1262 & - \\
\hline & Pitch & 1.788 & 1.775 & .0305 & .0305 \\
\hline \multirow[t]{2}{*}{11.7} & Plunge & 2.009 & - & .1392 & - \\
\hline & Pitch & 1.819 & 1.806 & .0176 & .0177 \\
\hline \multirow[t]{2}{*}{11.8} & Plunge & 1.984 & - & .1552 & - \\
\hline & Pitch & 1.844 & 1.831 & .0016 & .0017 \\
\hline \multirow[t]{2}{*}{11.9} & Plunge & 1.967 & - & .1713 & - \\
\hline & Pitch & - & - & -.0145 & -.0146 \\
\hline
\end{tabular}

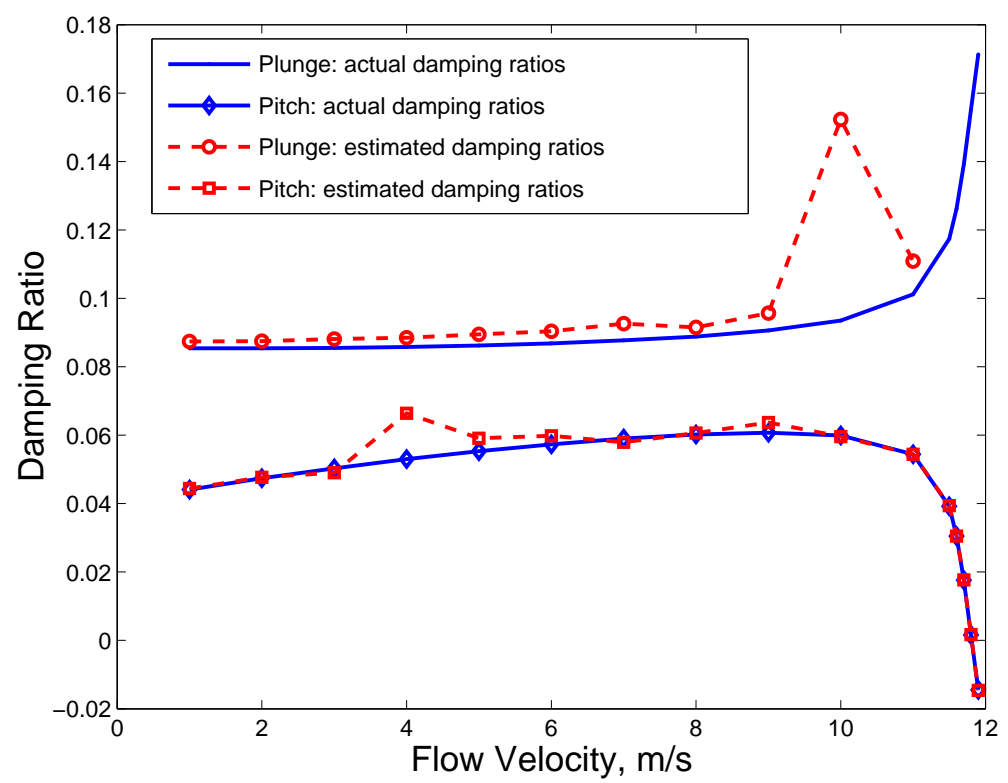

Figure 19. Actual and estimated damping ratios.

frequency range of each IMF is an option that could enhance the parameter estimation procedure. Also, even though parameters for both modes were estimated using a single IMF, the same results would generally not be obtained from an analysis of the impulse response function without the benefit of EMD. Although the IMF used in the analysis 
contains contributions from two modes, only one mode is present in the signal at any point in time. In contrast, the impulse response function may contain contributions from both modes over the same time period. This example demonstrates some potential benefits of the HHT for extracting modal parameters from aeroelastic flight data.

\section{Conclusions}

Application of the Hilbert-Huang algorithm for system signal decompositions, studying the effect of enhancements such as local/on-line behavior, understanding filtering properties, and especially for investigating correlations between input-output and between system sensors in terms of instantaneous properties, is revealed. System input-output signal analysis is introduced to characterize the time-varying amplitude and frequency components of multiple data channels, including input-to-output and distributed sensors, in terms of the intrinsic mode functions (IMFs) of the Hilbert-Huang transform (HHT). Significant departures from Fourier and other time-frequency or time-scale wavelet approaches are exposed. In these respects, this paper attempts to show how the HHT behaves in a sometimes nonintuitive and subtle manner in the analysis of F/A-18 Active Aeroelastic Wing (AAW) aircraft aeroelastic flight test data. Online stability analyses and modal identification are also presented. Examples are given using aeroelastic test data from the F/A-18 Active Aeroelastic Wing aircraft, an Aerostructures Test Wing, and pitch-plunge simulation.

An objective signal-adaptive basis function derivation, the Hilbert-Huang algorithm yields intrinsic mode functions giving instantaneous frequencies as functions of time that permit identification of imbedded structures. There is a multiresolution quality in the empirical mode decomposition process which even deals with intermittency by allowing multiple time-scales within an intrinsic mode function, but not allowing a similar time-scale simultaneously with other IMFs. System identification in the IMF sub-component environment is a practical endeavor in the domain of multiresolution system identification. It should be noted that the idea of exploiting local properties for signal analysis applies to spatial data as well as temporal data with frequency and scale (translation and duration) variations. From the idea of empiquency to describe oscillations in images based on extrema points there are potential applications for general time-space-frequency-scale signal processing.

Modern intelligent control and integrated aerostructures require control feedback signal processing congnizant of system stability and health. Time-varying linear or nonlinear modal characteristics derived from flight data are all within the realm of HHT. Further research will investigate these issues and HHT connections between localized instantaneous dynamics, health diagnostics, and global system stability and performance for monitoring and prediction.

\section{References}

${ }^{1}$ Bendat, J. S. and A.G. Piersol, Random Data Analysis and Measurement Procedures, John Wiley \& Sons, Inc., Chap. 12-13, 2000.

${ }^{2}$ Buresti, G., G. Lombardi, and J. Bellazzini, "On the analysis of fluctuating velocity signals through methods based on the wavelet and Hilbert transforms," Chaos, Solitons and Fractals, 20(2004), pp. 149-158.

${ }^{3}$ Chen, Q., N. Huang, S. Riemenschneider, and Y. Xu, "A B-spline approach for empirical mode decompositions," Advances in Computational Mathematics, 2004.

${ }^{4}$ Cohen, L., “Time-Frequency Distributions - A Review," Proceedings of the IEEE, 77(7), 1989, pp. 941-981.

${ }^{5}$ Flandrin, P., Time-Frequency/Time-Scale Analysis, Academic Press, 1999.

${ }^{6}$ Flandrin, P., G. Rilling, and P. Gonçalvès, "Empirical Mode Decomposition as a Filter Bank," IEEE Signal Processing Letters, 11(2), Feb 2004, pp. 112-114.

${ }^{7}$ Flandrin, P. and P. Gonçalvès, "Empirical Mode Decompositions as Data-Driven Wavelet-Like Expansions," accepted for publication in International Journal of Wavelets, Multiresolution and Information Processing, 2005.

${ }^{8}$ Huang, N. E., Z. Shen, S.R. Long, M.C. Wu, H.H. Shih, Q. Zheng, N-C Yen, C.C. Tung, and H.H. Liu, "The empirical mode decomposition and the Hilbert spectrum for nonlinear and non-stationary time series analysis," Proc. Royal Society London A, 454(1998), pp. 903-995.

${ }^{9}$ Huang, N. E., Z. Shen, and S.R. Long, "A New View of Nonlinear Water Waves: The Hilbert Spectrum," Annual Review of Fluid Mechanics, 31(1999), pp. 417-457.

${ }^{10}$ Huang, N. E., M-L.C. Liu, S.R. Long, S.S.P. Shen, W. Qu, P. Gloersen, and K.L. Fan, "A confidence limit for the empirical mode decomposition and Hilbert spectral analysis," Proc. Royal Society London A, 459(2003), pp. 2317-2345.

${ }^{11}$ Jha, R., F. Yan, and G. Ahmadi, "Energy-Frequency-Time Analysis of Structural Vibrations using Hibert-Huang Transform," AIAA-20041975.

${ }^{12}$ Lind, R., D.F. Voracek, R. Truax, T. Doyle, S. Potter, and M. Brenner, "A flight test to demonstrate flutter and evaluate the flutterometer," The Aeronautical Journal of the Royal Aeronautical Society, Oct 2003.

28 of 29 
${ }^{13}$ Linderhed, A., "2D empirical mode decompositions in the spirit of image compression," Wavelet and Independent Component Analysis Applications IX, SPIE Proceedings, 4738(2002), pp. 1-8.

${ }^{14}$ Linderhed, A., "Image compression based on empirical mode decomposition," Proc. of SSAB 04 Symp. on Image Analysis, Uppsala, Sweden, Mar 11-12, 2004, pp. 110-113.

${ }^{15}$ Liu, B., S. Riemenschneider, and Y. Xu, "Gearbox fault diagnosis using empirical mode decomposition and Hilbert spectrum," submitted for publication in Mechanical Systems and Signal Processing, 2004.

${ }^{16}$ Liu, Z. and S. Peng, "Boundary Processing of Bidimensional EMD Using Texture Synthesis," IEEE Signal Processing Letters, 12(1), Jan 2005, pp. 33-36.

${ }^{17}$ Loughlin, P. J. and L. Cohen, "The Uncertainty Principle: Global, Local, or Both?," IEEE Transactions on Signal Processing, 52(5), May 2004, pp. 1218-1227.

${ }^{18}$ Pendleton, E., D. Bessette, P. Field, G. Miller, and K. Griffin, "Active Aeroelastic Wing Flight Research Program: Technical Program and Model Analytical Development," AIAA Journal of Aircraft, 37(4), Jul-Aug 2000, pp. 554-561.

${ }^{19}$ Poolla, K., P. Khargonekar, A. Tikku, J. Krause, and K. Nagpal, “A Time-Domain Approach to Model Validation," IEEE Transactions on Automatic Control, 39(5), May 1994, pp. 951-959.

${ }^{20}$ Rilling, G., P. Flandrin, and P. Gonçalvès, "On Empirical Mode Decomposition and its algorithms," IEEE-EURASIP Workshop on Nonlinear Signal and Image Processing, NSIP-03, Grado, Italy, 2003.

${ }^{21}$ Sharpley, R. C. and V. Vatchev, "Analysis of the Intrinsic Mode Functions," for publication in Constructive Approximations, 2005.

${ }^{22}$ Vatchev, V., Intrinsic Mode Functions and the Hilbert Transform, Ph.D thesis, Dept of Mathematics, U. of South Carolina, 2004.

${ }^{23}$ Worden, K. and G.R. Tomlinson, Nonlinearity in Structural Dynamics: Detection, Identification, and Modeling, Institute of Physics Publishing Ltd, Bristol and Philadelphia, 2001.

${ }^{24}$ Wu, Z., N.E. Huang, "A study of the characteristics of white noise using the empirical mode decomposition method," Proc. Royal Society London A, 460 (2004), pp. 1597-1611.

${ }^{25}$ Yang, B. and C.S. Suh, "Interpretation of crack-induced rotor non-linear response using instantaneous frequency," Mechanical Systems and Signal Processing, 18(2004), pp. 491-513.

${ }^{26}$ Yang, J. N., Y. Lei, S. Pan, and N. Huang, "System identification of linear structures based on Hilbert-Huang spectral analysis. Part 1: Normal modes," Earthquake Engineering and Structural Dynamics, 32(2003), pp. 1443-1467.

${ }^{27}$ Yang, J. N., Y. Lei, S. Pan, and N. Huang, "System identification of linear structures based on Hilbert-Huang spectral analysis. Part 2: Complex modes," Earthquake Engineering and Structural Dynamics, 32(2003), pp. 1533-1554.

${ }^{28}$ Yang, J. N., Y. Lei, S. Pan, and N. Huang, "Identification of Natural Frequencies and Dampings of In Situ Tall Buildings Using Ambient Wind Vibration Data," Journal of Engineering Mechanics, 130(1), May 2004, pp. 1-8.

${ }^{29}$ Yang, J. N., Y. Lei, S. Pan, and N. Huang, "Hilbert-Huang Based Approach for Structural Damage Detection," Journal of Engineering Mechanics, 130(1), 2004, pp. 85-95.

${ }^{30}$ Zhou, K., with J. Doyle, Essentials of Robust Control, Prentice Hall, Upper Saddle River, New Jersey, 1998. 